\title{
Efficacy of Ovsynch Program on Reproductive Performance in Dairy Cattle: A Meta-Analysis
}

\author{
A. R. Rabiee, ${ }^{1}$ I. J. Lean, ${ }^{1}$ and M. A. Stevenson ${ }^{2}$ \\ ${ }^{1}$ Bovine Research Australasia, PO Box 660, Camden 2570, NSW, Australia \\ ${ }^{2}$ EpiCentre, Institute of Veterinary, Animal, and Biomedical Sciences, Massey University, \\ Palmerston North, New Zealand
}

\section{ABSTRACT}

The efficacy of the Ovsynch program in improving conception and pregnancy rates was compared with untreated controls and other synchrony programs in lactating dairy cows. This meta-analysis examined 71 treatment and control comparisons extracted from 53 research papers. Programs evaluated included Ovsynch, natural breeding, single, double, or triple prostaglandin injections, Select Synch, Heat Synch, and modified Ovsynch.

Pregnancy rates for Ovsynch programs did not differ significantly from those with natural breeding programs [predicted Bayesian relative risk $(R R)=1.04$, $95 \%$ Bayesian credible interval $=0.36$ to 3.23]. Results of Ovsynch vs. $\mathrm{PGF}_{2 \alpha}$ programs showed that the risk of conception (predicted Bayesian RR $=0.89$, 95\% Bayesian credible interval $=0.31$ to 2.64 ), and pregnancy rates predicted Bayesian $R R=1.11$, 95\% Bayesian credible interval $=0.61$ to 2.13 ) did not differ significantly between the Ovsynch group and cows in the $\mathrm{PGF}_{2 \alpha}$ group. Comparisons between Ovsynch and Select Synch demonstrated that the risk of conception (predicted Bayesian RR $=0.94,95 \%$ Bayesian credible interval $=0.52$ to 1.59 ), and pregnancy rates (predicted Bayesian $\mathrm{RR}=1.08,95 \%$ Bayesian credible interval $=$ 0.38 to 3.09 ) did not differ significantly between the 2 groups. Examination of Ovsynch vs. modified Ovsynch programs showed that the risk of pregnancy in cows synchronized with modified Ovsynch was similar to those treated with Ovsynch (predicted Bayesian RR = $0.89,95 \%$ Bayesian credible interval $=0.71$ to 1.12 ).

Meta-analyses identified that the conception and pregnancy rates obtained with the prostaglandin, Select Synch, and modified Ovsynch (including presynch and CoSynch) programs were comparable with the Ovsynch program. Modifications to the Ovsynch program such as presynchronization and timed artificial insemi-

Received December 19, 2004.

Accepted March 28, 2005.

Corresponding author: A. R. Rabiee; e-mail: ahmadr@dairydocs. com.au. nation at the time of second GnRH injection (CoSynch) may be an alternative for reproductive management of dairy herds where detection of estrus is less than optimal. The findings of this study demonstrate that the Ovsynch program could benefit dairy operations because it allows for timed artificial insemination of lactating cows without detection of estrus. There was, however, little or no significant improvement in pregnancy rates using Ovsynch over other programs and the costs of labor and hormone administration should be considered when selecting this form of reproductive technology for routine use.

(Key words: Ovsynch, dairy cattle, meta-analysis, Bayesian statistics)

Abbreviation key: $\mathbf{C I}=$ confidence or credible interval, ECP = estradiol cypionate, $\mathbf{M C M C}=$ Markov chain Monte Carlo, $\mathbf{R R}=$ relative risk, $\mathbf{T A I}=$ timed artificial insemination.

\section{INTRODUCTION}

Reproductive efficiency is one of the key components of a profitable dairy system. The physiological and environmental stresses of high milk production, inadequate nutrient intake, low body condition, and intensive management systems impair reproductive performance in dairy cattle (Butler, 2000; Thatcher et al., 2000). Major limiting factors for reproductive performance on many dairy farms are sensitive and specific estrus detection. Estrus detection rates in dairy herds are often low $(<50 \%)$ (Radostits et al., 1994; Quaife, 1995), and even when observation follows $\mathrm{PGF}_{2 \alpha}$-induced luteolysis for estrus synchronization, low rates of estrus detection persist. Breeding on observed estrus is more effective in achieving conception than is timed AI (TAI) after $\mathrm{PGF}_{2 \alpha}$ administration (Fetrow and Blanchard, 1987; Stevenson et al., 1987).

The variability in onset of ovulation after the end of a synchrony will determine whether an animal can be inseminated at prearranged times. To achieve maximum precision in ovulation synchrony, it is necessary to have a recently selected dominant follicle present by the completion of the treatment program. New wave 
emergence needs to be synchronized during treatment, because both the stage of follicular wave and the duration of dominance cause variation in the duration of the follicular phase. Gonadotropin-releasing hormone has been used to cause predictable new wave emergence in some cattle synchronization protocols, that is, either in combination with $\mathrm{PGF}_{2 \alpha}$ (Pursley et al., 1995, 1997a,b; Twagiramungu et al., 1995; Schmitt et al., 1996) or as part of a progesterone regimen (Ryan et al., 1995).

Systematic breeding programs aim to optimize reproductive management on the dairy farm by eliminating the need to detect estrus before breeding (DeJarnette et al., 2001). Incorporation of ovulation synchronization in dairy herd reproductive management programs allows producers to minimize labor requirements for estrus detection while improving overall reproductive performance (Pankowski et al., 1995). New technology has advanced the development of systems to improve pregnancy rates by synchronizing estrus and follicle development with regression of the corpus luteum, precisely controlling the time of ovulation to provide a fixed timed insemination and improve embryo survival (Thatcher et al., 2000, 2001a,b, 2002).

Several synchrony programs have been developed in recent years based on timed insemination. One of these programs, Ovsynch (Pursley et al., 1997a,b), is claimed to be more successful for the insemination of cows at fixed time for first service. The Ovsynch program consists of 2 injections of GnRH, $7 \mathrm{~d}$ before, and $48 \mathrm{~h}$ after an injection of $\mathrm{PGF}_{2 \alpha}$. Cows are inseminated 16 to 25 $\mathrm{h}$ after the second injection of GnRH. This system synchronizes follicle maturation with regression of the corpus luteum before the GnRH-induced ovulation and timed insemination. Several studies during the past few years have compared the Ovsynch program with other synchrony programs such as double $\mathrm{PGF}_{2 \alpha}$ (Pursley et al., 1997a; de la Sota et al., 1998; Keister et al., 1999; Stevenson et al., 1996, 1999, 2000; Cartmill et al., 2001a), progesterone-based programs (Geary et al., 1998; Williams et al., 2002), Select Synch (Burke et al., 1996; Stevenson et al., 1999, 2000; Cartmill et al., 2001b), Heat Synch (Bartolome et al., 2002; Pancarci et al., 2002), and natural breeding (Pursley et al., 1997b; Aréchiga et al., 1998; Keister et al., 1999; Cordoba and Fricke, 2001).

Meta-analysis is a technique for combining data from several trials, and is particularly useful when there is conflict in the literature and when sample sizes of individual studies are insufficient to detect a statistically significant result (L'Abbe et al., 1987; Peto, 1987). The objective of this study was to use meta-analytical methods to evaluate the efficacy of the Ovsynch program in improving conception and pregnancy rates compared with other synchrony programs in lactating dairy cows. This is an epidemiological approach to evaluate the efficacy of synchrony programs currently used in commercial dairy farms around the world. It is not the intention of this paper to provide physiologic or pharmacologic explanations for the success or failure of a particular program, as there are several comprehensive reviews on Ovsynch and other synchrony programs that provide these details (Thatcher et al., 2000; 2001a,b; 2002).

\section{MATERIALS AND METHODS}

All English-language papers and abstracts published between 1995 and 2003 that investigated the efficacy of the Ovsynch program on estrous cycle synchrony and conception and pregnancy rates in lactating dairy cows were identified by a computerized literature search (Commonwealth Agricultural Bureau, Biological Abstracts, PubMed, Scirus, and Sciencedirect), library searches of relevant journals, and a review of citations in identified review papers. Only those papers that met specified inclusion criteria were used for analysis. Because the primary objective of this study was to evaluate the efficacy of the Ovsynch program, all studies that compared Ovsynch with other programs in randomized trials with positive or negative controls were considered.

\section{Synchrony Programs}

The synchrony programs investigated and compared in this study are described below.

Ovsynch (GnRH-PGF $\boldsymbol{F}_{2 \alpha^{-}} \boldsymbol{G n R H}$ ). Gonadotropin-releasing hormone $(\mathrm{GnRH})$ injection followed by an injection of $\mathrm{PGF}_{2 \alpha} 7 \mathrm{~d}$ later, and a second $\mathrm{GnRH}$ injection given $48 \mathrm{~h}$ after $\mathrm{PGF}_{2 \alpha}$.

Natural breeding. Animals visually monitored for estrus and insemination on detection of estrus; or standard reproductive management for each trial, which consisted of mating using natural service bulls, or AI after detection of estrus aided by the use of Kamar heatmount detectors (Immucell, Portland, OR) or tail paint. Some programs included the occasional use of $\mathrm{PGF}_{2 \alpha}$ and $\mathrm{GnRH}$ when determined to be appropriate by the herd veterinarian or manager.

$\boldsymbol{P} \boldsymbol{G F}_{2 \alpha}$. Single, double, and triple $\mathrm{PGF}_{2 \alpha}$ injection 11 to $14 \mathrm{~d}$ apart, followed by insemination at the subsequently detected estrus that may have followed each injection.

Select Synch (GnRH-PGF $\left.\boldsymbol{F}_{2 \alpha}\right)$. Gonadotropin-releasing hormone injection followed in $7 \mathrm{~d}$ with an injection of $\mathrm{PGF}_{2 \alpha}$.

Heat Synch (GnRH-PGF $\left.F_{2 \alpha}-\boldsymbol{E C P}\right)$. Gonadotropinreleasing hormone injection followed in $7 \mathrm{~d}$ by an injec- 
tion of $\mathrm{PGF}_{2 \alpha}$ and estradiol cypionate (ECP) injection given $24 \mathrm{~h}$ after $\mathrm{PGF}_{2 \alpha}$.

Modified Ovsynch. Includes presynch Ovsynch (1 or 2 injections of $\mathrm{PGF}_{2 \alpha}$ given $14 \mathrm{~d}$ apart, with the first injection of the Ovsynch given 12 to $14 \mathrm{~d}$ after the second $\mathrm{PGF}_{2 \alpha}$ or injections of $\mathrm{PGF}_{2 \alpha}$ and $\mathrm{GnRH} 7 \mathrm{~d}$ apart), or presynchrony using controlled internal drug release insertion $7 \mathrm{~d}$ before the first injection of the Ovsynch program, and other minor modifications such as insemination at the time of second GnRH injection (CoSynch) and varying doses of $\mathrm{GnRH}$ and $\mathrm{PGF}_{2 \alpha}$.

We defined pregnancy rate as the proportion of treated or control cows that became pregnant by the date after intervention defined for each trial by those researchers. We defined conception rate as the proportion of inseminated cows only that became pregnant in either group. Overall pregnancy rate was the proportion of cows that became pregnant for cows that entered the trial by the end of breeding season or program as defined for each trial.

\section{Trials Included/Excluded in the Study}

Trials were included or excluded in the study based on criteria developed by the first 2 authors. Trials included in the analysis were those in which: (1) an adequate description of the randomization process was provided, (2) animals were lactating at the time of study enrollment, and (3) sufficient data were included to determine rates of conception and pregnancy. Of all the papers describing the results of an Ovsynch program, only those trials that met the defined inclusion criteria were included in the meta-analyses described. Published data in journal papers were crosschecked with conference papers to avoid repetition in presented data. All trials that compared Ovsynch with other synchrony programs, including those not considered for the metaanalysis, are listed in Table 1. Trials were ineligible for inclusion in the analysis if they were not randomized clinical trials $(n=6)$, if the trial was compromised with supplementary treatment $(n=8)$, if the subjects were beef $(n=5)$ or dairy heifers $(n=7)$ rather lactating dairy cows, or if the paper provided insufficient data on the variables being measured $(n=12)$.

\section{Extracted Data}

Three reproductive outcomes were commonly reported and selected for evaluation in this meta-analysis. Data extracted from suitable trials were number of cows, conception and pregnancy rates. Other information that was extracted included; study design, number of cows in the treatment and control groups, negative or positive controls, the treatment protocol including the dose and days of $\mathrm{GnRH}$ and $\mathrm{PGF}_{2 \alpha}$ administration, breeding type (at detected estrus or TAI). Where the papers provided results from different farms, animals, or series of studies, these were considered separate trials. In some cases, information was only extracted from first or second round of synchrony programs. Results reported for each outcome were examined and, where appropriate, pooled to give a summary estimate of relative risk and a $95 \%$ credible interval (CI).

\section{Statistical Analyses}

Conception rates, individual service pregnancy rates and overall pregnancy rates from all trials selected for inclusion were used to calculate the relative risk (RR) of conception and pregnancy in Ovsynch-treated cows, compared with other synchrony programs. Variations among trial-level relative risks were assessed using a $\chi^{2}$ test of heterogeneity. In this case, the null hypothesis was that the effect of treatment was the same across $k$ trials and the null hypothesis was rejected if the heterogeneity test statistic was greater than a critical value that separated the upper $10 \%$ of a $\chi^{2}$ distribution with $(k-1)$ degrees of freedom. We used an $\alpha$ level of 0.10 because of the relatively poor power of the $\chi^{2}$ test to detect heterogeneity among small numbers of trials (Egger and Smith, 2001).

Heterogeneity of results among trials was quantified using the $I^{2}$ statistic, (Higgins et al., 2003). The $I^{2}$ statistic describes the percentage of total variation across studies that is due to heterogeneity rather than chance. Where $Q$ is the $\chi^{2}$ heterogeneity statistic and $\mathrm{k}$ is the number of trials, $I^{2}$ was calculated as:

$$
I^{2}(\%)=\frac{Q-(k-1)}{Q} \times 100
$$

Uncertainty intervals for $I^{2}$ (dependent on $Q$ and $k$ ) were calculated using the method described by Higgins and Thompson (2002). Negative values of $I^{2}$ were put equal to zero, consequently $I^{2}$ lay between 0 and $100 \%$. A value greater than $50 \%$ may be considered substantial heterogeneity.

To estimate the effect of an Ovsynch synchrony program compared with other programs on reproductive outcomes, we used Bayesian meta-analysis models (Domenici et al., 1999). Here, the observed number of events in the control group of each trial, $\mathrm{O}_{\mathrm{i}}^{\mathrm{C}}$, was assumed to be from a binomial distribution defined by parameters $\pi_{\mathrm{i}}^{\mathrm{C}}$ (representing the underlying probability of a positive reproductive outcome in the control group) and $n_{i}^{\mathrm{C}}$ (representing the number of subjects in the control group). The observed number of events in the treat- 
Table 1. Treatment protocols for Ovsynch, natural breeding, $\mathrm{PGF}_{2 \alpha}$, Select Synch, Heat Synch, and modified Ovsynch in lactating dairy cows and heifers.

\begin{tabular}{|c|c|c|c|c|c|c|c|}
\hline \multirow[b]{2}{*}{ Reference } & \multirow[b]{2}{*}{ Trial } & \multicolumn{2}{|c|}{ Treatment protocol ${ }^{1}$} & \multicolumn{2}{|c|}{$\begin{array}{l}\text { Hormone } e^{2} \text { dose for } \\
\text { Ovsynch }\end{array}$} & \multicolumn{2}{|c|}{ Breeding protocol } \\
\hline & & Control & Ovsynch & $\mathrm{PGF}_{2 \alpha}$ & GnRH & Control & Ovsynch \\
\hline $\begin{array}{l}\text { Stevenson et al. } \\
\text { (1996) }\end{array}$ & $\begin{array}{l}\text { Heifers } \\
\text { and cows }\end{array}$ & $2 \times \mathrm{PGF}_{2 \alpha}(14 \mathrm{~d}$ apart $)$ & $\begin{array}{l}\text { G-P-G } \\
0-7-9\end{array}$ & $\begin{array}{l}25 \mathrm{mg} \\
\text { Lutalyse }\end{array}$ & $\begin{array}{l}100 \mu \mathrm{g} \\
\text { Cystorelin }\end{array}$ & AI at detected estrus & $\begin{array}{l}\mathrm{TAI}^{4} 18-19 \mathrm{~h} \\
\text { later }\end{array}$ \\
\hline $\begin{array}{l}\text { Burke et al. } \\
(1996)\end{array}$ & & $\begin{array}{l}\text { G-P } \\
0-7\end{array}$ & $\begin{array}{l}\text { G-P-G } \\
0-7-9\end{array}$ & $\begin{array}{l}25 \mathrm{mg} \\
\text { Lutalyse }\end{array}$ & $\begin{array}{l}100 \mu \mathrm{g} \\
\text { Buserelin }\end{array}$ & $\mathrm{AI}$ at detected estrus & $\begin{array}{l}\text { TAI } 16-20 \mathrm{~h} \\
\text { later }\end{array}$ \\
\hline $\begin{array}{l}\text { Schmitt et al. } \\
\text { (1996) }\end{array}$ & $\begin{array}{l}\text { Trial } 1 \\
\text { (Heifers) } \\
\text { Trial } 2 \\
\text { (Heifers) }\end{array}$ & $\begin{array}{l}\text { G-P } \\
0-7 \\
\text { G-P } \\
0-7\end{array}$ & $\begin{array}{l}\text { G-P-G } \\
0-7-8 \\
\text { G-P-G } \\
0-7-9\end{array}$ & $\begin{array}{l}25 \mathrm{mg} \\
\text { Lutalyse } \\
25 \mathrm{mg} \\
\text { Lutalyse }\end{array}$ & $\begin{array}{l}8 \mu \mathrm{g} \\
\text { Buserelin } \\
8 \mu \mathrm{g} \\
\text { Buserelin }\end{array}$ & $\begin{array}{l}\text { AI } 0-12 \mathrm{~h} \text { after detected } \\
\text { estrus } \\
\text { AI } 0-12 \mathrm{~h} \text { after detected } \\
\text { estrus }\end{array}$ & $\begin{array}{l}\text { TAI } 15 \mathrm{~h} \\
\text { later } \\
\text { TAI } 15 \mathrm{~h} \\
\text { later }\end{array}$ \\
\hline $\begin{array}{l}\text { Pursley et al. } \\
\text { (1997a) }\end{array}$ & $\begin{array}{l}\text { Heifers } \\
\text { and cows }\end{array}$ & $\begin{array}{l}\text { Spontaneous estrus } \\
\text { (visual monitoring and } \\
\text { occasional use of } \mathrm{PGF}_{2 \alpha} \\
\text { and GnRH) }\end{array}$ & $\begin{array}{l}\text { G-P-G } \\
0-7-9\end{array}$ & $\begin{array}{l}25 \mathrm{mg} \\
\text { Lutalyse }\end{array}$ & $\begin{array}{l}100 \mu \mathrm{g} \\
\text { Cystorelin }\end{array}$ & $\mathrm{AI}$ at detected estrus & $\begin{array}{l}\text { TAI } 16-20 \mathrm{~h} \\
\text { later }\end{array}$ \\
\hline $\begin{array}{l}\text { Pursley et al. } \\
\text { (1997b) }\end{array}$ & & $3 \times \mathrm{PGF}_{2 \alpha}(14 \mathrm{~d}$ apart $)$ & $\begin{array}{l}\text { G-P-G } \\
0-7-9\end{array}$ & $\begin{array}{l}25 \mathrm{mg} \\
\text { Lutalyse }\end{array}$ & $\begin{array}{l}100 \mu \mathrm{g} \\
\text { Cystorelin }\end{array}$ & $\begin{array}{l}\text { AI at detected estrus after } \\
\text { each injection and TAI } \\
(72-80 \mathrm{~h}) \text { after } 3 \mathrm{rd}\end{array}$ & $\begin{array}{l}\text { TAI } 16-20 \mathrm{~h} \\
\text { later }\end{array}$ \\
\hline $\begin{array}{l}\text { de la Sota et al. } \\
\text { (1998) }\end{array}$ & & $1 \times \mathrm{PGF}_{2 \alpha}$ & $\begin{array}{l}\text { G-P-G } \\
0-7-9\end{array}$ & $\begin{array}{l}25 \mathrm{mg} \\
\text { Lutalyse }\end{array}$ & $\begin{array}{l}8 \mu \mathrm{g} \\
\text { Buserelin }\end{array}$ & $\mathrm{AI}$ at detected estrus & $\begin{array}{l}\text { TAI } 16 \mathrm{~h} \\
\text { later }\end{array}$ \\
\hline $\begin{array}{l}\text { Fricke et al. } \\
\text { (1998) }\end{array}$ & & $\begin{array}{l}\text { G }(50 \mu \mathrm{g})-\mathrm{P}-\mathrm{G}(50 \mu \mathrm{g}) \\
0-7-9\end{array}$ & $\begin{array}{l}\text { G-P-G } \\
0-7-9\end{array}$ & $\begin{array}{l}25 \mathrm{mg} \\
\text { Lutalyse }\end{array}$ & $\begin{array}{l}100 \mu \mathrm{g} \\
\text { Cystorelin }\end{array}$ & TAI 12-18 h later & $\begin{array}{l}\text { TAI } 12-18 \mathrm{~h} \\
\text { later }\end{array}$ \\
\hline $\begin{array}{l}\text { Britt and Gaska } \\
\text { (1998) }\end{array}$ & & $1 \times \mathrm{PGF}_{2 \alpha}$ & $\begin{array}{l}\text { G-P-G } \\
0-7-8.5\end{array}$ & $\begin{array}{l}25 \mathrm{mg} \\
\text { Lutalyse }\end{array}$ & $\begin{array}{l}100 \mu \mathrm{g} \\
\text { Cystorelin }\end{array}$ & $\mathrm{AI}$ at detected estrus & $\begin{array}{l}\text { TAI } 17 \mathrm{~h} \\
\text { later }\end{array}$ \\
\hline $\begin{array}{l}\text { Aréachiga et al. } \\
\text { (1998) }\end{array}$ & & $\begin{array}{l}\text { Spontaneous estrus } \\
\text { (visual monitoring and } \\
\text { estrus detection using } \\
\text { K-mar) }\end{array}$ & $\begin{array}{l}\text { G-P-G } \\
0-7-9\end{array}$ & $\begin{array}{l}25 \mathrm{mg} \\
\text { Lutalyse }\end{array}$ & $\begin{array}{l}8 \mu \mathrm{g} \\
\text { Receptal }\end{array}$ & $\begin{array}{l}\text { AI at detected estrus or a } \\
\text { day after }\end{array}$ & $\begin{array}{l}\text { TAI } 15-25 \mathrm{~h} \\
\text { later }\end{array}$ \\
\hline $\begin{array}{l}\text { Keister et al. } \\
\text { (1999) }\end{array}$ & $\begin{array}{l}\text { Trial } 1 \\
\text { Trial } 2 \\
\text { Trial } 3\end{array}$ & $\begin{array}{l}2 \times \mathrm{PGF}_{2 \alpha} \\
\text { (14 d apart) } \\
\text { Spontaneous estrus } \\
\text { (visual monitoring) } \\
\text { G-P-G (initiated } 7 \mathrm{~d} \\
\text { after estrus) } \\
0-7-9\end{array}$ & $\begin{array}{l}\text { G-P-G } \\
0-7-9 \\
\text { G-P-G } \\
0-7-9 \\
\text { G-P-G } \\
0-7-9\end{array}$ & $\begin{array}{l}25 \mathrm{mg} \\
\text { Lutalyse } \\
25 \mathrm{mg} \\
\text { Lutalyse } \\
25 \mathrm{mg} \\
\text { Lutalyse }\end{array}$ & $\begin{array}{l}100 \mu \mathrm{g} \\
\text { Cystorelin } \\
100 \mu \mathrm{g} \\
\text { Cystorelin } \\
100 \mu \mathrm{g} \\
\text { Cystorelin }\end{array}$ & $\begin{array}{l}\text { AI at detected estrus after } \\
\text { 2nd injection } \\
\text { AI at detected estrus } \\
\text { TAI } 16-20 \mathrm{~h} \text { later }\end{array}$ & $\begin{array}{l}\text { TAI } 16-20 \mathrm{~h} \\
\text { later } \\
\text { TAI } 16-20 \mathrm{~h} \\
\text { later } \\
\text { TAI } 16-20 \mathrm{~h} \\
\text { later }\end{array}$ \\
\hline $\begin{array}{l}\text { Stevenson et al. } \\
\text { (1999) }\end{array}$ & Trial 2 & $\begin{array}{l}\text { G-P } \\
0-7 \\
\text { G-P } \\
0-7\end{array}$ & $\begin{array}{l}\text { G-P-G } \\
0-7-8.5 \\
\text { G-P-G } \\
0-7-9\end{array}$ & $\begin{array}{l}25 \mathrm{mg} \\
\text { Lutalyse } \\
25 \mathrm{mg} \\
\text { Lutalyse }\end{array}$ & $\begin{array}{l}8 \mu \mathrm{g} \\
\text { Buserelin } \\
100 \mu \mathrm{g} \\
\text { Cystorelin }\end{array}$ & $\begin{array}{l}\text { AI } 10-14 \mathrm{~h} \text { after detected } \\
\text { estrus } \\
\text { AI } 10-14 \mathrm{~h} \text { after detected } \\
\text { estrus }\end{array}$ & $\begin{array}{l}\text { TAI } 16-18 \mathrm{~h} \\
\text { later } \\
\text { TAI } 16-20 \mathrm{~h} \\
\text { later }\end{array}$ \\
\hline $\begin{array}{l}\text { Stevenson et al. } \\
\text { (1999) }\end{array}$ & Trial 1 & $2 \times \mathrm{PGF}_{2 \alpha}(14 \mathrm{~d}$ apart $)$ & $\begin{array}{l}\text { G-P-G } \\
0-7-9\end{array}$ & $\begin{array}{l}25 \mathrm{mg} \\
\text { Lutalyse }\end{array}$ & $\begin{array}{l}100 \mu \mathrm{g} \\
\text { Cystorelin }\end{array}$ & $\begin{array}{l}\text { AI at detected estrus after } \\
\text { 2nd injection and TAI }(80 \mathrm{~h})\end{array}$ & $\begin{array}{l}\text { TAI } \\
16-18 \mathrm{~h} \\
\text { later }\end{array}$ \\
\hline & Trial 2 & $\begin{array}{l}2 \times \mathrm{PGF}_{2 \alpha}(14 \mathrm{~d} \text { apart })+ \\
\text { GnRH on d } 15\end{array}$ & $\begin{array}{l}\text { G-P-G } \\
0-7-9\end{array}$ & $\begin{array}{l}25 \mathrm{mg} \\
\text { Lutalyse }\end{array}$ & $\begin{array}{l}100 \mu \mathrm{g} \\
\text { Cystorelin }\end{array}$ & TAI 16-18 h later & $\begin{array}{l}\text { TAI } 16-18 \mathrm{~h} \\
\text { later }\end{array}$ \\
\hline $\begin{array}{l}\text { Mawhinney et al. } \\
\text { (1999) }\end{array}$ & & $\begin{array}{l}\text { Spontaneous estrus } \\
\text { (visual monitoring) }\end{array}$ & $\begin{array}{l}\text { G-P-G } \\
0-7-9\end{array}$ & $\begin{array}{l}25 \mathrm{mg} \\
\text { Lutalyse }\end{array}$ & $\begin{array}{l}100 \mu \mathrm{g} \\
\text { Fertagyl }\end{array}$ & $\mathrm{AI}$ at detected estrus & $\begin{array}{l}\text { TAI } 12-20 \mathrm{~h} \\
\text { later }\end{array}$ \\
\hline Mialot et al. (1999) & Trial 1 & $\begin{array}{l}1 \text { or } 2 \times \mathrm{PGF}_{2 \alpha} \\
(13 \mathrm{~d} \text { apart })\end{array}$ & $\begin{array}{l}\text { G-P-G } \\
0-7-9\end{array}$ & $\begin{array}{l}25 \mathrm{mg} \\
\text { Enzaprost }\end{array}$ & $\begin{array}{l}100 \mu \mathrm{g} \\
\text { Cystorelin }\end{array}$ & $\begin{array}{l}\text { AI at detected estrus after } \\
1 \text { st injection, or } 72 \text { and } 96 \mathrm{~h} \\
\text { after 2nd injection }\end{array}$ & $\begin{array}{l}\text { TAI } 20-24 \mathrm{~h} \\
\text { later }\end{array}$ \\
\hline & Trial 2 & $\begin{array}{l}1 \text { or } 2 \times \mathrm{PGF}_{2 \alpha} \\
(13 \mathrm{~d} \text { apart })\end{array}$ & $\begin{array}{l}\text { G-P-G } \\
0-7-9\end{array}$ & $\begin{array}{l}25 \mathrm{mg} \\
\text { Enzaprost }\end{array}$ & $\begin{array}{l}100 \mu \mathrm{g} \\
\text { Cystorelin }\end{array}$ & $\begin{array}{l}\mathrm{AI} \text { at detected estrus after } \\
1 \text { st and } 2 \text { nd injection }\end{array}$ & $\begin{array}{l}\text { AI at estrus } \\
\text { after d } 0 \text {, or } \\
\text { TAI } 20-24 \mathrm{~h} \\
\text { later }\end{array}$ \\
\hline
\end{tabular}


Table 1 (Continued). Treatment protocols for Ovsynch, natural breeding, $\mathrm{PGF}_{2 \alpha}$, Select Synch, Heat Synch, and modified Ovsynch in lactating dairy cows and heifers.

\begin{tabular}{|c|c|c|c|c|c|c|c|}
\hline \multirow[b]{2}{*}{ Reference } & \multirow[b]{2}{*}{ Trial } & \multicolumn{2}{|c|}{ Treatment protocol ${ }^{1}$} & \multicolumn{2}{|c|}{$\begin{array}{l}\text { Hormone }{ }^{2} \text { dose for } \\
\text { Ovsynch }\end{array}$} & \multicolumn{2}{|c|}{ Breeding protocol } \\
\hline & & Control & Ovsynch & $\mathrm{PGF}_{2 \alpha}$ & GnRH & Control & Ovsynch \\
\hline \multirow[t]{2}{*}{$\begin{array}{l}\text { Stevenson et al. } \\
(2000)\end{array}$} & $\begin{array}{l}\text { Trial } 1 \\
\text { (Heifers) }\end{array}$ & $\begin{array}{l}2 \times \mathrm{PGF}_{2 \alpha} \\
(14 \mathrm{~d} \text { apart })\end{array}$ & $\begin{array}{l}\text { G-P-G } \\
0-7-8 \text { or } 9\end{array}$ & $\begin{array}{l}25 \mathrm{mg} \\
\text { Lutalyse }\end{array}$ & $\begin{array}{l}100 \mu \mathrm{g} \\
\text { Cystorelin }\end{array}$ & $\begin{array}{l}\text { AI } 8-16 \mathrm{~h} \text { after detected } \\
\text { estrus and TAI }(72-80 \mathrm{~h}) \\
\text { after } 2 \text { nd injection }\end{array}$ & $\begin{array}{l}\text { TAI } 15-25 \mathrm{~h} \\
\text { later }\end{array}$ \\
\hline & $\begin{array}{l}\text { Trial } 2 \\
\text { (Heifers) }\end{array}$ & $\begin{array}{l}\text { G-P } \\
0-6 \text { or } 7\end{array}$ & $\begin{array}{l}\text { G-P-G } \\
0-7-8 \text { or } 9\end{array}$ & $\begin{array}{l}25 \mathrm{mg} \\
\text { Lutalyse }\end{array}$ & $\begin{array}{l}50 \mu \mathrm{g} \\
\text { Cystorelin }\end{array}$ & $\begin{array}{l}\text { AI } 8-16 \mathrm{~h} \text { after detected } \\
\text { estrus }\end{array}$ & $\begin{array}{l}\text { TAI } 15-25 \mathrm{~h} \\
\text { later }\end{array}$ \\
\hline Jemmeson (2000) & & $2 \times \mathrm{PGF}_{2 \alpha}(14 \mathrm{~d}$ apart $)$ & $\begin{array}{l}\text { G-P-G } \\
0-7-9\end{array}$ & $\begin{array}{l}25 \mathrm{mg} \\
\text { Lutalyse }\end{array}$ & $\begin{array}{l}100 \mu \mathrm{g} \\
\text { Cystorelin }\end{array}$ & $\mathrm{AI}$ at detected estrus & $\begin{array}{l}\text { TAI } 16-20 \mathrm{~h} \\
\text { later }\end{array}$ \\
\hline \multirow[t]{2}{*}{$\begin{array}{l}\text { Amiridis et al. } \\
(2000)\end{array}$} & Trial 1 & $\begin{array}{l}\text { G-P-hCG (3000 IU) } \\
0-7-9\end{array}$ & $\begin{array}{l}\text { G-P-G } \\
0-7-9\end{array}$ & $\begin{array}{l}15 \mathrm{mg} \\
\text { Pronilen }\end{array}$ & $\begin{array}{l}200 \mu \mathrm{g} \\
\text { Fertolin }\end{array}$ & TAI 14-18 h later & $\begin{array}{l}\text { TAI } 14-18 \mathrm{~h} \\
\text { later }\end{array}$ \\
\hline & Trial 2 & $2 \times \mathrm{PGF}_{2 \alpha}(11 \mathrm{~d}$ apart $)$ & $\begin{array}{l}\text { G-P-G } \\
0-7-9\end{array}$ & $\begin{array}{l}15 \mathrm{mg} \\
\text { Pronilen }\end{array}$ & $\begin{array}{l}200 \mu \mathrm{g} \\
\text { Fertolin }\end{array}$ & $\begin{array}{l}\text { TAI } 72 \text { and } 96 \mathrm{~h} \text { after } \\
\text { 2nd injection }\end{array}$ & $\begin{array}{l}\text { TAI } 14-18 \mathrm{~h} \\
\text { later }\end{array}$ \\
\hline \multirow[t]{3}{*}{ Jobst et al. (2000) } & Trial 1 & $2 \times \mathrm{PGF}_{2 \alpha}(14 \mathrm{~d}$ apart $)$ & $\begin{array}{l}\text { G-P-G } \\
0-7-9\end{array}$ & $\begin{array}{l}500 \mu \mathrm{g} \\
\text { Estrumate }\end{array}$ & $\begin{array}{l}86 \mu \mathrm{g} \\
\text { Fertagyl }\end{array}$ & $\begin{array}{l}\text { AI at detected estrus after } \\
\text { each injection or AI } \\
\text { within } 14 \mathrm{~h} \text { after estrus } \\
\text { with HeatWatch }\end{array}$ & $\begin{array}{l}\text { TAI } 16-18 \mathrm{~h} \\
\text { later }\end{array}$ \\
\hline & Trial 2 & $\begin{array}{l}\text { G-P } \\
0-7\end{array}$ & $\begin{array}{l}\text { G-P-G } \\
0-7-9\end{array}$ & $\begin{array}{l}500 \mu \mathrm{g} \\
\text { Estrumate }\end{array}$ & $\begin{array}{l}86 \mu \mathrm{g} \\
\text { Fertagyl }\end{array}$ & $\mathrm{AI}$ at detected estrus & $\begin{array}{l}\text { TAI } 16-18 \mathrm{~h} \\
\text { later }\end{array}$ \\
\hline & Trial 3 & $\begin{array}{l}\text { Spontaneous estrus } \\
\text { detected by visual } \\
\text { observation or HeatWatch }\end{array}$ & $\begin{array}{l}\text { G-P-G } \\
0-7-9\end{array}$ & $\begin{array}{l}500 \mu \mathrm{g} \\
\text { Estrumate }\end{array}$ & $\begin{array}{l}86 \mu \mathrm{g} \\
\text { Fertagyl }\end{array}$ & $14 \mathrm{~h}$ after onset of estrus & $\begin{array}{l}\text { TAI } 16-18 \mathrm{~h} \\
\text { later }\end{array}$ \\
\hline $\begin{array}{l}\text { Tallam et al. } \\
\text { (2001) }\end{array}$ & & $2 \times \mathrm{PGF}_{2 \alpha}(14 \mathrm{~d}$ apart $)$ & $\begin{array}{l}\text { G-P-G +hCG } \\
0-7-9-14\end{array}$ & $\begin{array}{l}25 \mathrm{mg} \\
\text { Lutalyse }\end{array}$ & $\begin{array}{l}50 \mu \mathrm{g} \\
\text { Cystorelin }\end{array}$ & $\begin{array}{l}\text { AI at detected estrus after } \\
\text { each injection }\end{array}$ & $\begin{array}{l}\text { TAI } 22 \mathrm{~h} \\
\text { later }\end{array}$ \\
\hline $\begin{array}{l}\text { Tenhagen et al. } \\
\text { 2001) }\end{array}$ & & $2 \times \mathrm{PGF}_{2 \alpha}(14 \mathrm{~d}$ apart $)$ & $\begin{array}{l}\text { G-P-G } \\
0-7-9\end{array}$ & $\begin{array}{l}0.75 \mathrm{mg} \\
\text { Iliren }\end{array}$ & $\begin{array}{l}20 \mu \mathrm{g} \\
\text { Buserelin }\end{array}$ & $\begin{array}{l}\text { TAI } 66-90 \mathrm{~h} \text { after } 2 \mathrm{nd} \\
\text { injection }\end{array}$ & $\begin{array}{l}\text { TAI } 16-20 \mathrm{~h} \\
\text { later }\end{array}$ \\
\hline $\begin{array}{l}\text { Cartmill et al. } \\
(2001 b)\end{array}$ & Trial 1 & $\begin{array}{l}2 \times \mathrm{PGF}_{2 \alpha}(12 \mathrm{~d} \text { apart }) \\
+\mathrm{GnRH} \text { on } \mathrm{d} 14\end{array}$ & $\begin{array}{l}\text { G-P-G } \\
0-7-9\end{array}$ & $\begin{array}{l}25 \mathrm{mg} \\
\text { Lutalyse }\end{array}$ & $\begin{array}{l}100 \mu \mathrm{g} \\
\text { Cystorelin }\end{array}$ & TAI $16-20 \mathrm{~h}$ later & $\begin{array}{l}\text { TAI } 16-20 \mathrm{~h} \\
\text { later }\end{array}$ \\
\hline $\begin{array}{l}\text { Cartmill et al. } \\
\text { (2001a) }\end{array}$ & & $\begin{array}{l}\text { G-P } \\
0-7\end{array}$ & $\begin{array}{l}\text { G-P-G } \\
0-7-9\end{array}$ & $\begin{array}{l}25 \mathrm{mg} \\
\text { Lutalyse }\end{array}$ & $\begin{array}{l}100 \mu \mathrm{g} \\
\text { Fertagyl }\end{array}$ & $\mathrm{AI}$ at detected estrus & $\begin{array}{l}\text { TAI } 16-19 \mathrm{~h} \\
\text { later }\end{array}$ \\
\hline \multirow[t]{2}{*}{$\begin{array}{l}\text { DeJarnette et al. } \\
\text { (2001) }\end{array}$} & Trial 1 & $\begin{array}{l}\text { G-P-AI (+ GnRH in cows } \\
\text { not in estrus) } \\
0-7-10\end{array}$ & $\begin{array}{l}\text { G-P-G } \\
0-7-9\end{array}$ & $\begin{array}{l}25 \mathrm{mg} \\
\text { Lutalyse }\end{array}$ & $\begin{array}{l}100 \mu \mathrm{g} \\
\text { Cystorelin }\end{array}$ & TAI $72 \mathrm{~h}$ after 2 nd $P$ & $\begin{array}{l}\text { TAI } 16-18 \mathrm{~h} \\
\text { later }\end{array}$ \\
\hline & Trial 2 & $\begin{array}{l}\text { G-P-AI (+ GnRH in cows } \\
\text { not in estrus) } \\
0-7-10\end{array}$ & $\begin{array}{l}\text { G-P-G } \\
0-7-9\end{array}$ & $\begin{array}{l}25 \mathrm{mg} \\
\text { Lutalyse }\end{array}$ & $\begin{array}{l}100 \mu \mathrm{g} \\
\text { Cystorelin }\end{array}$ & TAI $72 \mathrm{~h}$ after second $\mathrm{P}$ & $\begin{array}{l}\text { TAI } 16-18 \mathrm{~h} \\
\text { later }\end{array}$ \\
\hline \multirow[t]{2}{*}{$\begin{array}{l}\text { Cordoba and Fricke } \\
\text { (2001) }\end{array}$} & Trial 1 & $\begin{array}{l}\text { P-G-P-G } \\
(-22)-0-7-9\end{array}$ & $\begin{array}{l}\text { G-P-G } \\
0-7-9\end{array}$ & $\begin{array}{l}25 \mathrm{mg} \\
\text { Lutalyse }\end{array}$ & $\begin{array}{l}100 \mu \mathrm{g} \\
\text { Cystorelin }\end{array}$ & TAI 16-18 h later & $\begin{array}{l}\text { TAI } 16-18 \mathrm{~h} \\
\text { later }\end{array}$ \\
\hline & Trial 2 & $\begin{array}{l}\text { Estrus detection using } \\
\text { K-mar }\end{array}$ & $\begin{array}{l}\text { G-P-G } \\
0-7-9\end{array}$ & $\begin{array}{l}25 \mathrm{mg} \\
\text { Lutalyse }\end{array}$ & $\begin{array}{l}50 \mu \mathrm{g} \\
\text { Cystorelin }\end{array}$ & $\begin{array}{l}\text { Natural service (bull) } \\
\text { and AI at detected estrus }\end{array}$ & $\begin{array}{l}\text { TAI } 17-25 \mathrm{~h} \\
\text { later }\end{array}$ \\
\hline Fricke (2001) & & $\begin{array}{l}(\mathrm{G}+\mathrm{CIDR})^{4}-\mathrm{P}-\mathrm{G} \\
0-7-9\end{array}$ & $\begin{array}{l}\text { G-P-G } \\
0-7-9\end{array}$ & $\begin{array}{l}25 \mathrm{mg} \\
\text { Lutalyse }\end{array}$ & $\begin{array}{l}50-100 \mu \mathrm{g} \\
\text { Cystorelin }\end{array}$ & TAI 12-18 h later & $\begin{array}{l}\text { TAI } 12-18 \mathrm{~h} \\
\text { later }\end{array}$ \\
\hline $\begin{array}{l}\text { Moreira et al. } \\
(2001)\end{array}$ & & $\begin{array}{l}2 \times \mathrm{PGF}_{2 \alpha}(14 \mathrm{~d})-\mathrm{G}-\mathrm{P}-\mathrm{G} \\
0-7-9\end{array}$ & $\begin{array}{l}\text { G-P-G } \\
0-7-9\end{array}$ & $\begin{array}{l}40 \mathrm{mg} \\
\text { Lutalyse }\end{array}$ & $\begin{array}{l}50-100 \mu \mathrm{g} \\
\text { Cystorelin }\end{array}$ & TAI $16-20 \mathrm{~h}$ later & $\begin{array}{l}\text { TAI } 16-20 \mathrm{~h} \\
\text { later }\end{array}$ \\
\hline \multirow[t]{2}{*}{$\begin{array}{l}\text { Cordoba and Fricke } \\
\text { (2001) }\end{array}$} & Trial 1 & $\begin{array}{l}\text { Estrus detection using } \\
\text { K-mar }\end{array}$ & $\begin{array}{l}\text { G-P-G } \\
0-7-9\end{array}$ & $\begin{array}{l}25 \mathrm{mg} \\
\text { Lutalyse }\end{array}$ & $\begin{array}{l}50 \mu \mathrm{g} \\
\text { Cystorelin }\end{array}$ & $\begin{array}{l}\text { Natural service (bull) } \\
\text { and AI at detected estrus }\end{array}$ & $\begin{array}{l}\text { TAI } 17-25 \mathrm{~h} \\
\text { later }\end{array}$ \\
\hline & Trial 2 & $\begin{array}{l}\text { Estrus detection using } \\
\text { K-mar }\end{array}$ & $\begin{array}{l}\text { G-P-G } \\
0-7-9\end{array}$ & $\begin{array}{l}25 \mathrm{mg} \\
\text { Lutalyse }\end{array}$ & $\begin{array}{l}50 \mu \mathrm{g} \\
\text { Cystorelin }\end{array}$ & $\begin{array}{l}\text { Natural service (bull) } \\
\text { and AI at detected estrus }\end{array}$ & $\begin{array}{l}\text { TAI } 17-25 \mathrm{~h} \\
\text { later }\end{array}$ \\
\hline \multirow[t]{2}{*}{$\begin{array}{l}\text { Alnimer et al. } \\
(2002)\end{array}$} & Trial 1 & $2 \times \mathrm{PGF}_{2 \alpha}(14 \mathrm{~d}$ apart $)$ & $\begin{array}{l}\text { G-P-G } \\
0-7-9\end{array}$ & $\begin{array}{l}15 \mathrm{mg} \\
\text { Prosolvin }\end{array}$ & $\begin{array}{l}100 \mu \mathrm{g} \\
\text { Fertagyl }\end{array}$ & $\begin{array}{l}\text { AI at detected estrus } \\
\text { after 2nd injection }\end{array}$ & $\begin{array}{l}\text { TAI } 16-20 \mathrm{~h} \\
\text { later }\end{array}$ \\
\hline & Trial 2 & $1 \times \mathrm{PGF}_{2 \alpha}$ & $\begin{array}{l}\text { G-P-G } \\
0-7-9\end{array}$ & $\begin{array}{l}15 \mathrm{mg} \\
\text { Prosolvin }\end{array}$ & $\begin{array}{l}100 \mu \mathrm{g} \\
\text { Fertagyl }\end{array}$ & AI at detected estrus & $\begin{array}{l}\text { TAI } 16-20 \mathrm{~h} \\
\text { later }\end{array}$ \\
\hline
\end{tabular}


Table 1 (Continued). Treatment protocols for Ovsynch, natural breeding, PGF $_{2 \alpha}$, Select Synch, Heat Synch, and modified Ovsynch in lactating dairy cows and heifers.

\begin{tabular}{|c|c|c|c|c|c|c|c|}
\hline \multirow[b]{2}{*}{ Reference } & \multirow[b]{2}{*}{ Trial } & \multicolumn{2}{|c|}{ Treatment protocol ${ }^{1}$} & \multicolumn{2}{|c|}{$\begin{array}{c}\text { Hormone } e^{2} \text { dose for } \\
\text { Ovsynch }\end{array}$} & \multicolumn{2}{|c|}{ Breeding protocol } \\
\hline & & Control & Ovsynch & $\mathrm{PGF}_{2 \alpha}$ & GnRH & Control & Ovsynch \\
\hline $\begin{array}{l}\text { Cordoba and Fricke } \\
(2002)\end{array}$ & & Tail paint & $\begin{array}{l}\text { G-P-G } \\
0-7-9\end{array}$ & $\begin{array}{l}25 \mathrm{mg} \\
\text { Lutalyse }\end{array}$ & $\begin{array}{l}50 \mu \mathrm{g} \\
\text { Cystorelin }\end{array}$ & $\begin{array}{l}\text { AI based on removed } \\
\text { tail-paint }\end{array}$ & $\begin{array}{l}\text { TAI } 12-18 \mathrm{~h} \\
\text { later }\end{array}$ \\
\hline $\begin{array}{l}\text { Pancarci et al. } \\
\text { (2002) }\end{array}$ & & $\begin{array}{l}\text { G-P-ECP } \\
0-7-8\end{array}$ & $\begin{array}{l}\text { G-P-G } \\
0-7-9\end{array}$ & $\begin{array}{l}25 \mathrm{mg} \\
\text { Lutalyse }\end{array}$ & $\begin{array}{l}100 \mu \mathrm{g} \\
\text { Cystorelin }\end{array}$ & TAI $48 \mathrm{~h}$ later & $\begin{array}{l}\text { TAI } 16-18 \mathrm{~h} \\
\text { later }\end{array}$ \\
\hline $\begin{array}{l}\text { Bartolome et al. } \\
(2002)\end{array}$ & & $\begin{array}{l}\text { G-P-ECP } \\
0-7-8\end{array}$ & $\begin{array}{l}\text { G-P-G } \\
0-7-9\end{array}$ & $\begin{array}{l}25 \mathrm{mg} \\
\text { Lutalyse }\end{array}$ & $\begin{array}{l}100 \mu \mathrm{g} \\
\text { Cystorelin }\end{array}$ & TAI $48 \mathrm{~h}$ later & $\begin{array}{l}\text { TAI } 16 \mathrm{~h} \\
\text { later }\end{array}$ \\
\hline Kyoji et al. (2002) & & $\begin{array}{l}\text { G (50 mg)-P-G (50 mg) } \\
0-7-9\end{array}$ & $\begin{array}{l}\text { G-P-G } \\
0-7-9\end{array}$ & $\begin{array}{l}500 \mu \mathrm{g} \\
\text { Resipron-C }\end{array}$ & $\begin{array}{l}100 \mu \mathrm{g} \\
\text { Concelarl }\end{array}$ & TAI 16-19 h later & $\begin{array}{l}\text { TAI } 16-19 \mathrm{~h} \\
\text { later }\end{array}$ \\
\hline $\begin{array}{l}\text { Peters and Pursley } \\
(2002)\end{array}$ & & $\begin{array}{l}\text { P-G (preSynch): G-P-G } \\
-10-(-7) 0-7-9\end{array}$ & $\begin{array}{l}\text { G-P-G } \\
0-7-9\end{array}$ & $\begin{array}{l}25 \mathrm{mg} \\
\text { Lutalyse }\end{array}$ & $\begin{array}{l}100 \mu \mathrm{g} \\
\text { Cystorelin }\end{array}$ & TAI $16 \mathrm{~h}$ later & $\begin{array}{l}\text { TAI } 16 \mathrm{~h} \\
\text { later }\end{array}$ \\
\hline Lean et al. (2003) & & $2 \times \mathrm{PGF}_{2 \alpha}(11 \mathrm{~d}$ apart $)$ & $\begin{array}{l}\text { G-P-G } \\
0-7-9\end{array}$ & $\begin{array}{l}15 \mathrm{mg} \\
\text { Prosolvin }\end{array}$ & $\begin{array}{l}100 \mu \mathrm{g} \\
\text { Fertagyl }\end{array}$ & $\mathrm{AI}$ at detected estrus & $\begin{array}{l}\text { TAI } 17-21 \mathrm{~h} \\
\text { later }\end{array}$ \\
\hline $\begin{array}{l}\text { DeJarnette and } \\
\text { Marshall (2003) }\end{array}$ & $\begin{array}{l}\text { Trial } 1 \\
\text { Trial } 2\end{array}$ & $\begin{array}{l}\text { P-P (preSynch) + Cosynch } \\
-35-(-21)-0-7-9 \\
\text { P-G (preSynch) + Cosynch } \\
-35-(-21)-(-14)-0-7-9\end{array}$ & $\begin{array}{l}\text { G-P-G } \\
0-7-9 \\
\text { G-P-G } \\
0-7-9\end{array}$ & $\begin{array}{l}25 \mathrm{mg} \\
\text { Lutalyse } \\
25 \mathrm{mg} \\
\text { ProstaMate }\end{array}$ & $\begin{array}{l}100 \mu \mathrm{g} \\
\text { Cystorelin } \\
100 \mu \mathrm{g} \\
\text { Fertagyl }\end{array}$ & $\begin{array}{l}\text { TAI and GnRH 60-64 h } \\
\text { after } \mathrm{PGF}_{2 \alpha} \\
\text { TAI and GnRH 60-64 h } \\
\text { after } \mathrm{PGF}_{2 \alpha}\end{array}$ & $\begin{array}{l}\text { TAI } 12-16 \mathrm{~h} \\
\text { later } \\
\text { TAI } 12-16 \mathrm{~h} \\
\text { later }\end{array}$ \\
\hline
\end{tabular}

${ }^{1}$ Treatments: G-P-G $=$ GnRH-PGF ${ }_{2 \alpha}-\mathrm{GnRH}$; and 0-7-9 = days the injections were given; ECP = estradiol cypionate.

${ }^{2}$ Hormones: Lutalyse (Pharmacia Animal Health); Enzaprost (Sanofi); Estrumate (Bayer); Prosolvin (Intervet); ProstaMate (Phoenix Scientific Inc.); Pronilen (Intervet); Iliren (Intervet GmbH); Cysterolin (Merial Limited \& Sanofi Animal Health); Buserelin (Hoechst-Roussel, Agri-Vet); Fertolin (Intervet); Fertagyl (Intervet); human chorionic gonadotropin (Cholulon, Intervet); ECP (Pharmacia Corp); CIDR (InterAg, NZ); Concelarl (Takeda Cering Purau Animal Health, Tokyo, Japan); Resipron-C (Teikoku Hormones Mfg. Co. Ltd., Tokyo, Japan); Receptal (Hoechst-Roussel Agri-Vet Co., ${ }^{3}$ TAI = Timed artificial insemination.

${ }^{4} \mathrm{G}+\mathrm{CIDR}$ : This program consisted of a controlled internal drug release (CIDR) for $6 \mathrm{~d}$ at the time of the first GnRH injection and injection of PGF ${ }_{2 \alpha}$ at the time of $\mathrm{CIDR}_{\mathrm{N}}$ removal.

${ }^{5}$ Heat Synch $=$ a similar protocol to Select Synch plus an injection of $1 \mathrm{mg}$ ECP on d 10 and TAI. 
ment group, $\mathrm{O}_{\mathrm{i}}^{\mathrm{T}}$, was similarly defined by parameters, $\pi_{\mathrm{i}}^{\mathrm{T}}$ and $\mathrm{n}_{\mathrm{i}}^{\mathrm{T}}$ :

$$
\begin{aligned}
& O_{i}^{C} \sim \operatorname{binomial}\left(\pi_{i}^{C}, n_{i}^{C}\right) \\
& O_{i}^{T} \sim \operatorname{binomial}\left(\pi_{i}^{T}, n_{i}^{T}\right)
\end{aligned}
$$

Using a fixed-effects Bayesian approach, we assumed that the log of the relative risk of a positive reproductive outcome following treatment, $\log \left(\pi_{\mathrm{i}}^{\mathrm{T}} / \pi_{\mathrm{i}}^{\mathrm{C}}\right)$, followed a normal distribution with mean $\theta$ and variance proportional to the number of subjects enrolled in the trial. Within the Bayesian paradigm, an uninformed normal prior centered at zero was assigned to $\theta$.

To account for heterogeneity among trial results, the fixed-effects model was extended to a random-effects model where the $\log$ of $\pi_{\mathrm{i}}^{\mathrm{C}}$ was assumed a function of individual trial-level effects $\lambda_{\mathrm{i}}$ and the $\log$ of $\pi_{\mathrm{i}}^{\mathrm{T}}$ assumed to be a function of individual trial-level and treatment effects $\left(\lambda_{\mathrm{i}}+\theta_{\mathrm{i}}\right)$.

$$
\log \left(\frac{\pi_{i}^{T}}{\pi_{i}^{C}}\right)=\frac{\lambda_{i}+\theta_{i}}{\lambda_{i}}
$$

In this case, the effect of treatment, $\theta_{\mathrm{i}}$, was parameterized as having a normal distribution with mean $\mu$ and variance $\tau^{2}$. This allowed the effect of treatment observed across $i$ trials $\left(\theta_{\mathrm{i}}\right)$ to vary around a "true" mean $(\mu)$ with variance $\tau^{2}$. An uninformed normal prior centered at zero was used for $\mu$ and an uninformed inverse gamma distribution used for $\tau^{2}$. An uninformed normal prior centered at zero was assigned to the individual trial-level effect parameter, $\lambda_{\mathrm{i}}$.

To estimate values of the various parameters, a joint probability distribution was formed by combining the prior distributions with the likelihood, given the observed data. Markov chain Monte Carlo (MCMC) methods implemented within the WinBUGS package (Spiegelhalter et al., 2002) were used to obtain samples from the joint posterior distribution to estimate $\theta$ (in the case of the fixed-effects models) and $\mu$ and $\tau^{2}$ (in the case of the random effects models). Our aim was to provide summary measures of treatment effect relevant to clinical decision making; thus (in addition to reporting the credible intervals of the relative risk estimates for the fixed- and random effects models), summary measures of effect have been reported as the $95 \% \mathrm{CI}$ of the relative risk estimates derived from $\mu$ and $\tau^{2}$. In practical terms, this predicted distribution should be thought of as the relative risk estimate of a successful reproductive outcome when an Ovsynch or other synchrony programs are used in a future (or unobserved) trial (Parmigiani, 2002).
For the Bayesian analyses, the MCMC sampler was run for 40,000 iterations and the first 1000 burn-in samples were discarded. Convergence of the posterior sampling distribution was visually assessed using cumulative path plots and quantified using the Raftery and Lewis convergence diagnostic (Raftery and Lewis, 1992a,b). Posterior sample sizes were determined by running sufficient iterations to ensure that the MCMC standard error of the posterior means were at least one order of magnitude smaller than their posterior standard deviation. Parallel chains of the MCMC sampler were run using diverse initial values to ensure that convergence was achieved to the same distribution (Gelman, 1996).

Sensitivity to the distributional assumptions was assessed by assuming different distributions for the trial effects and comparing subsequent inferences. For example, a second series of analyses was conducted where the variance of treatment effect $\left(\tau^{2}\right)$ was assumed to arise from a uniform distribution. Within a model, we determined how sensitive the combined estimate was to any one trial. This was achieved by leaving one trial out, calculating the combined effect of the remaining trials, and comparing the results with the combined effect based on all trials.

Results of each model were summarized as forest plots. Here, each study was represented by a black square and a horizontal line, corresponding to the point estimate and the $95 \%$ CI of the individual trial-level relative risk. Pooled relative risk estimates (and their 95\% CI) for the fixed-effects, random effects, and prediction model were denoted by diamonds on the same plot.

\section{Publication Bias}

Funnel plots (Light and Pillemer, 1984) were constructed to identify the presence of publication bias for each outcome. For each study included, estimates of the precision were plotted on the horizontal axis and study standard error on the vertical axis. The nature of sample distribution means that the plot will be naturally funnel-shaped with an apex pointing upwards. If publication bias is present, the plot will have a deficiency, usually in the area occupied by small negative studies. It is based on the premise that the precision in estimating the treatment difference will increase as the sample size of the study increases (Whitehead, 2002).

\section{RESULTS}

This study examined 71 treatment and control comparisons extracted from 53 research papers of several synchrony programs in cycling and anestrous lactating 


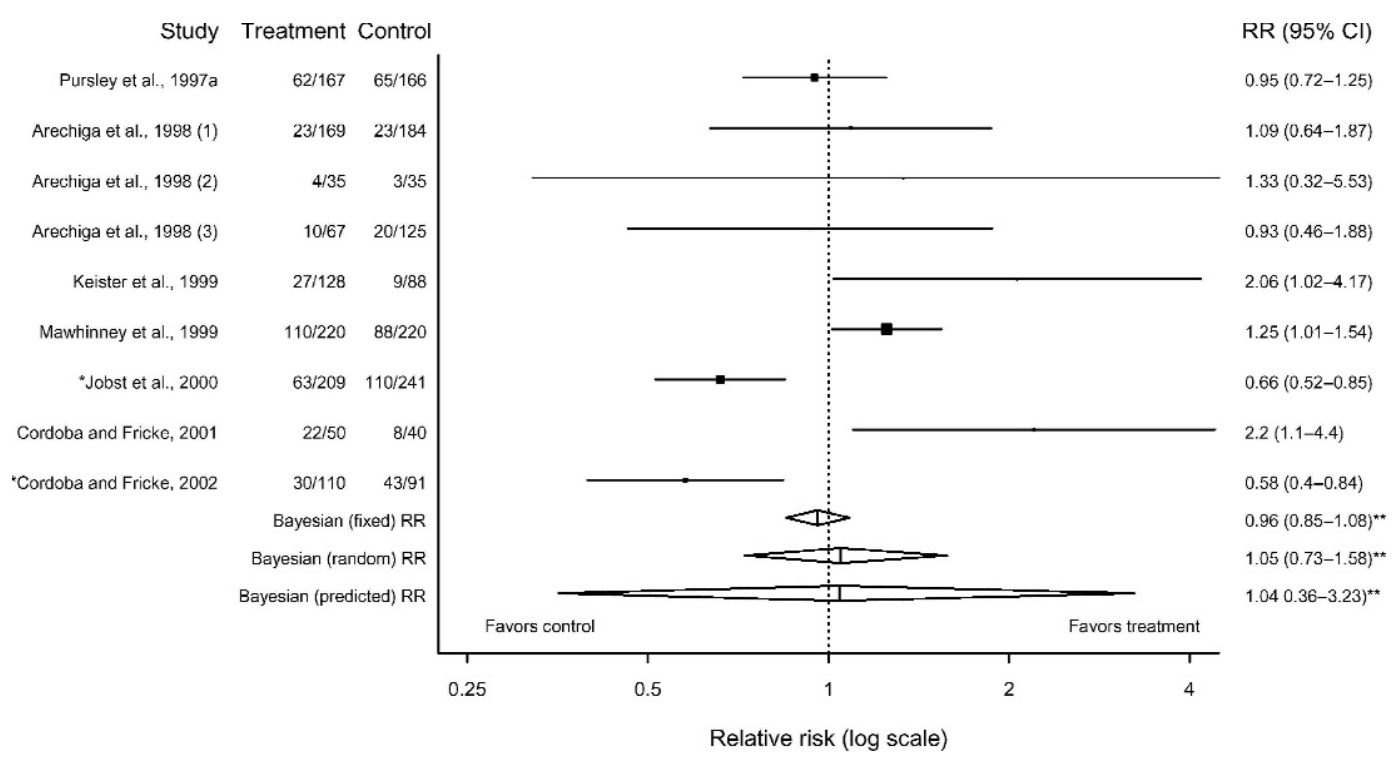

Figure 1. Relative risks (RR) [and their 95\% confidence interval (CI) for individual trials] determined from the results of 9 trials comparing pregnancy rates in Ovsynch and natural breeding programs. Box sizes are proportional to the inverse variance of the estimates. Summary estimates of treatment effects are shown using: 1) a Bayesian (fixed effects) approach, 2) a Bayesian (random effects) approach, and 3) the predicted distribution of relative risk estimates expected in a future trial and their Bayesian credible intervals. Test of heterogeneity: $I^{2}=$ 77 (56-88); test of heterogeneity following exclusion of studies marked with *: $I^{2}=34(0-72)$; ** 95\% Bayesian credible interval.

dairy cows. These synchrony programs included Ovsynch, natural breeding, $\mathrm{PGF}_{2 \alpha}$, Select Synch, Heat Synch, and modified Ovsynch.

Details of data extracted from each paper are presented in Table 1 . The treatment protocol in synchrony programs included the dose and days of GnRH and $\mathrm{PGF}_{2 \alpha}$ administration, duration of treatment, days of intravaginal controlled internal drug release insertion; and day, time, and type of insemination (at observed estrus or TAI) for each synchrony program.

Nine trials comparing Ovsynch with natural breeding extracted from 7 papers were used in the metaanalysis of conception rates in lactating dairy cows. A total of 2345 cows were used in these studies. Figure 1 gives the crude RR of conception and pregnancy rates for individual trials. Bayesian (pooled and predicted) $\mathrm{RR}$ are provided to summarize treatment effects across all included trials. The Ovsynch synchrony program did not produce a significant increase in pregnancy rate for all trials combined (predicted Bayesian $\mathrm{RR}=1.04 ; 95 \%$ Bayesian CI $=0.36$ to 3.23 ; Figure 1) and the results of this comparison were heterogeneous $\left(I^{2}=77 \%\right.$, uncertainty intervals $=56$ to $88 \%$, Table 2). Sensitivity analyses conducted following the exclusion of studies of Cordoba and Fricke (2002) and Jobst et al. (2000) showed that the results of remaining studies were homogeneous $\left(I^{2}=34 \%\right.$; uncertainty intervals $=0$ to $\left.72 \%\right)$.

Thirteen papers containing 17 trials on Ovsynch vs. $\mathrm{PGF}_{2 \alpha}$ programs were used in the meta-analysis of con- ception rate, pregnancy rate, and overall pregnancy rates in lactating dairy cows (Figures 2 to 4 ), representing 4970 cows. The risk of conception and pregnancy rates in the Ovsynch-treated group did not differ from those in the $\mathrm{PGF}_{2 \alpha}$ program (predicted Bayesian $\mathrm{RR}=$ 0.89; $95 \%$ Bayesian CI $=0.31$ to 2.64 , Figure 2, and predicted Bayesian RR $=1.11 ; 95 \%$ Bayesian $\mathrm{CI}=0.61$ to 2.13 , Figure 3 , respectively). Both fixed- and randomeffects models showed that the results of conception and pregnancy rates were heterogeneous (Table 2, Figures 2 and 3). The results of overall pregnancy rate were homogeneous $\left(I^{2}=45 \%\right.$, uncertainty intervals $=0$ to $77 \%$, Table 2).

Five trials on Ovsynch vs. Select Synch extracted from 4 papers were used in the meta-analysis of conception and pregnancy rates in lactating dairy cows. A total of 1534 cows were used in these studies. The risk of conception and pregnancy in cows synchronized with Ovsynch did not differ significantly when compared with those synchronized with Select Synch program (predicted Bayesian RR $=0.94,95 \%$ Bayesian $\mathrm{CI}=0.52$ to 1.59 , Figure 5 and predicted Bayesian $R R=1.08$; $95 \%$ Bayesian CI $=0.38$ to 3.09, Figure 6 , respectively). Both fixed- and random-effects models showed that the results of pregnancy trials on Ovsynch vs. Select Synch were heterogeneous $\left(I^{2}=82 \%\right.$, uncertainty intervals $=$ 60 to $92 \%$, Table 2). Sensitivity analyses conducted following the exclusion of pregnancy rate data in the stud- 
Table 2. Summary of pooled predicted Bayesian relative risks for conception and pregnancy rates for dairy cows treated with Ovsynch program compared with other breeding programs.

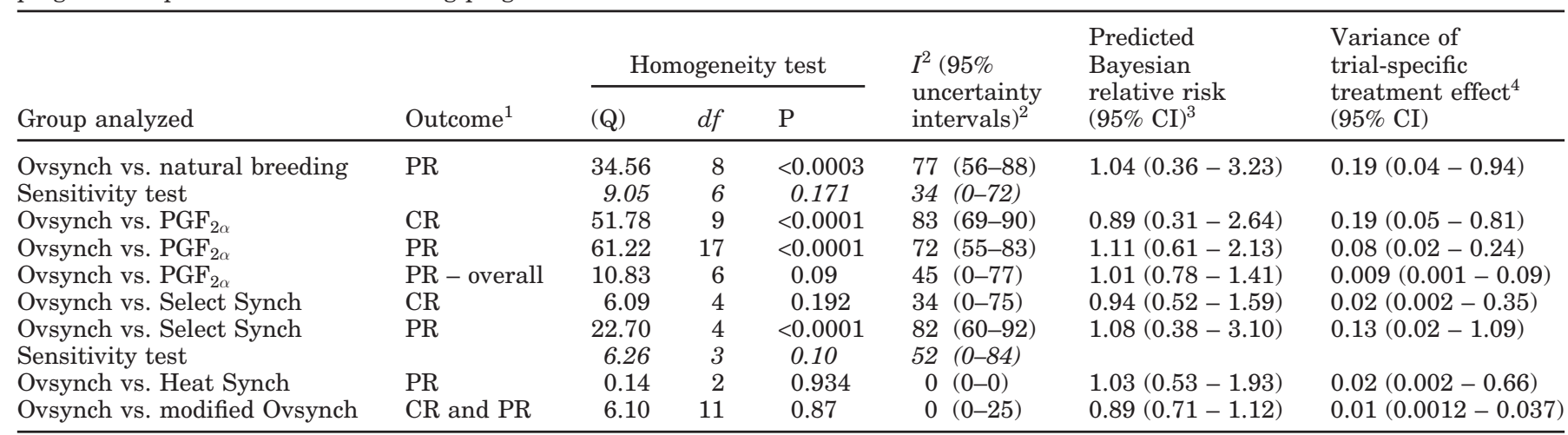

${ }^{1}$ Outcome: $\mathrm{PR}$ = pregnancy rate; $\mathrm{CR}=$ conception rate.

${ }^{2}$ Values of $I^{2}$ describe the percentage of total variation across studies that is due to heterogeneity rather than chance; the $95 \%$ uncertainty intervals are calculated as proposed by Higgins and Thompson (2002).

${ }^{3} \mathrm{CI}=$ Credible interval.

${ }^{4}$ Variance of trial-specific treatment effect $\left(\tau^{2}\right)$.

ies of Jobst et al. (2000) showed that the results of remaining studies were homogeneous (Table 2).

Data from 12 trials on Ovsynch vs. modified Ovsynch extracted from 9 papers were used in the meta-analysis of conception and pregnancy rates in lactating dairy cows. These trials represent 3324 cows. The risks of conception and pregnancy in cows synchronized with Ovsynch were comparable with those from the modified Ovsynch program (predicted Bayesian $R R=0.89,95 \%$
Bayesian CI $=0.71$ to 1.12 , Figure 7). Fixed- and random-effects models showed that the results of studies on Ovsynch vs. modified Ovsynch were homogeneous $\left(I^{2}=0 \%\right.$, uncertainty intervals $=0$ to $25 \%$, Table 2$)$.

Summary conception and pregnancy rates for all synchrony programs are presented in Table 2. This includes the pooled RR, predicted RR (Bayesian models), 95\% CI, variance of trial-specific treatment effect and its $95 \% \mathrm{CI}$, heterogeneity $\chi^{2}$, degrees of freedom (df),

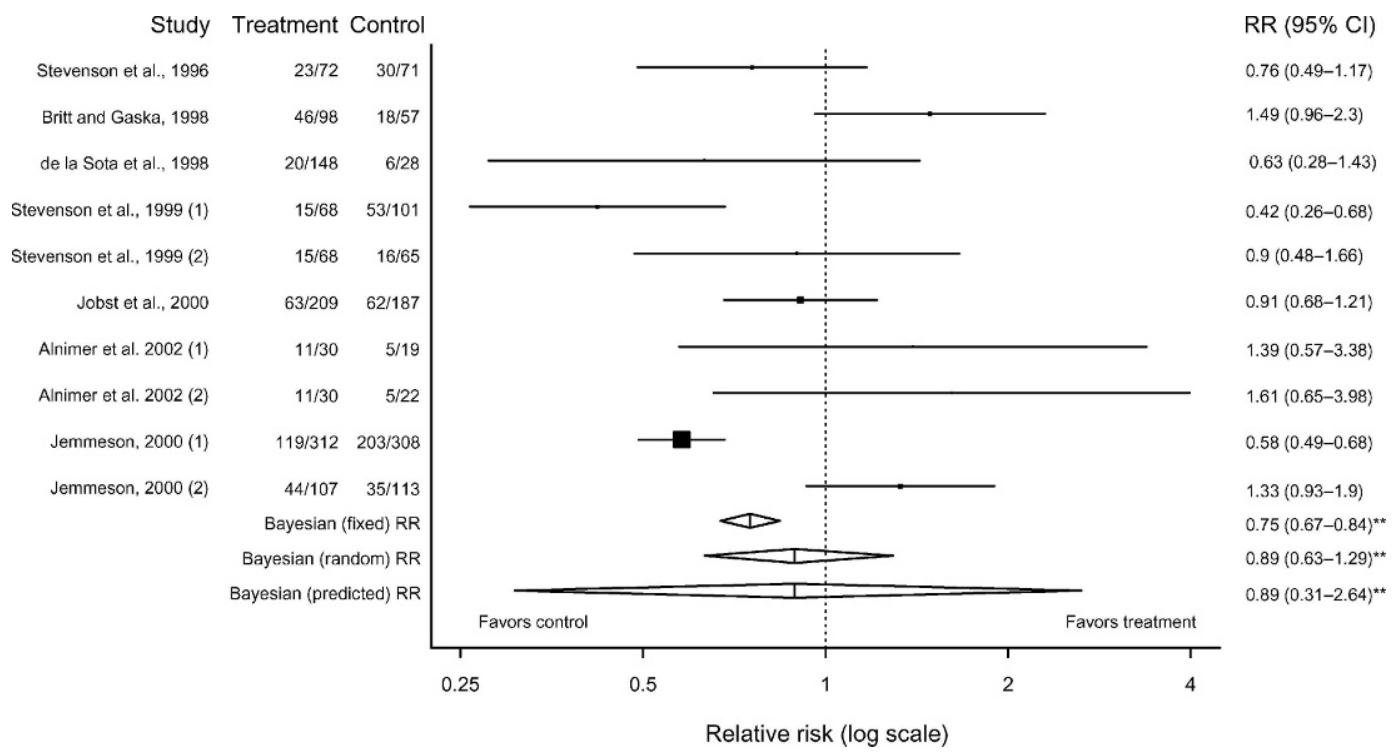

Figure 2. Relative risks (RR) [and their 95\% confidence interval (CI) for individual trials] determined from the results of 10 trials comparing conception rates in Ovsynch and prostaglandin programs. Box sizes are proportional to the inverse variance of the estimates. Summary estimates of treatment effects are shown using: 1) a Bayesian (fixed effects) approach, 2) a Bayesian (random effects) approach, and 3) the predicted distribution of relative risk estimates expected in a future trial and their Bayesian credible intervals. Test of heterogeneity: $I^{2}=83(69-90) ; * * 95 \%$ Bayesian credible interval. 


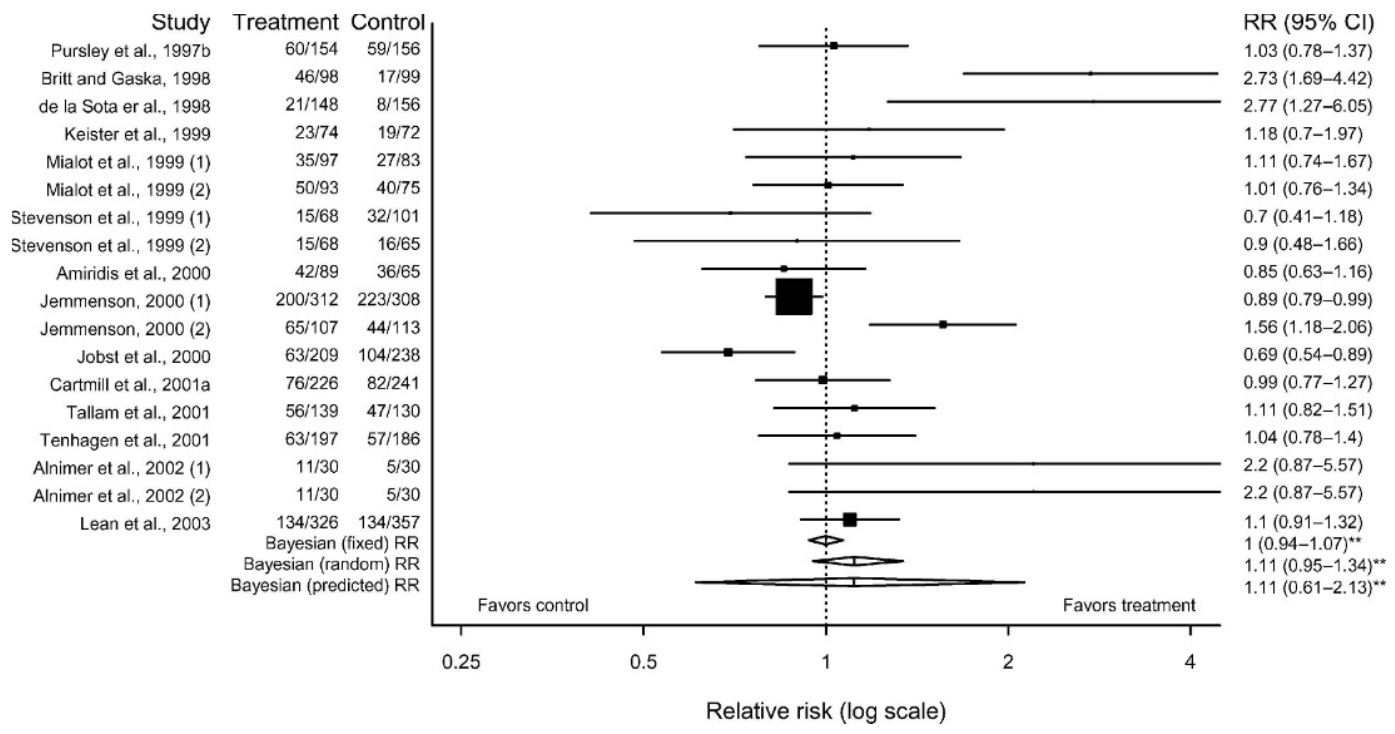

Figure 3. Relative risks (RR) [and their 95\% confidence interval (CI) for individual trials] determined from the results of 18 trials comparing pregnancy rates in Ovsynch and prostaglandin programs. Box sizes are proportional to the inverse variance of the estimates. Summary estimates of treatment effects are shown using: 1) a Bayesian (fixed effects) approach, 2) a Bayesian (random effects) approach, and 3) the predicted distribution of relative risk estimates expected in a future trial and their Bayesian credible intervals. Test of heterogeneity: $I^{2}=72(55-83) ; * * 95 \%$ Bayesian credible interval.

significance of $\chi^{2}$, and $I^{2}$ and its uncertainty intervals. Results of sensitivity analysis on selected synchrony program comparisons are presented in Table 2.

Funnel plots (Figures 8 and 9) of 2 comparisons were constructed to demonstrate use of funnel plots to assess publication bias. Figure 8 was constructed for Ovsynch vs. natural breeding; the plot was not symmetrical and the small number of studies made it difficult to conclude whether publication bias was present. Figure 9 was constructed for Ovsynch vs. modified Ovsynch, and it

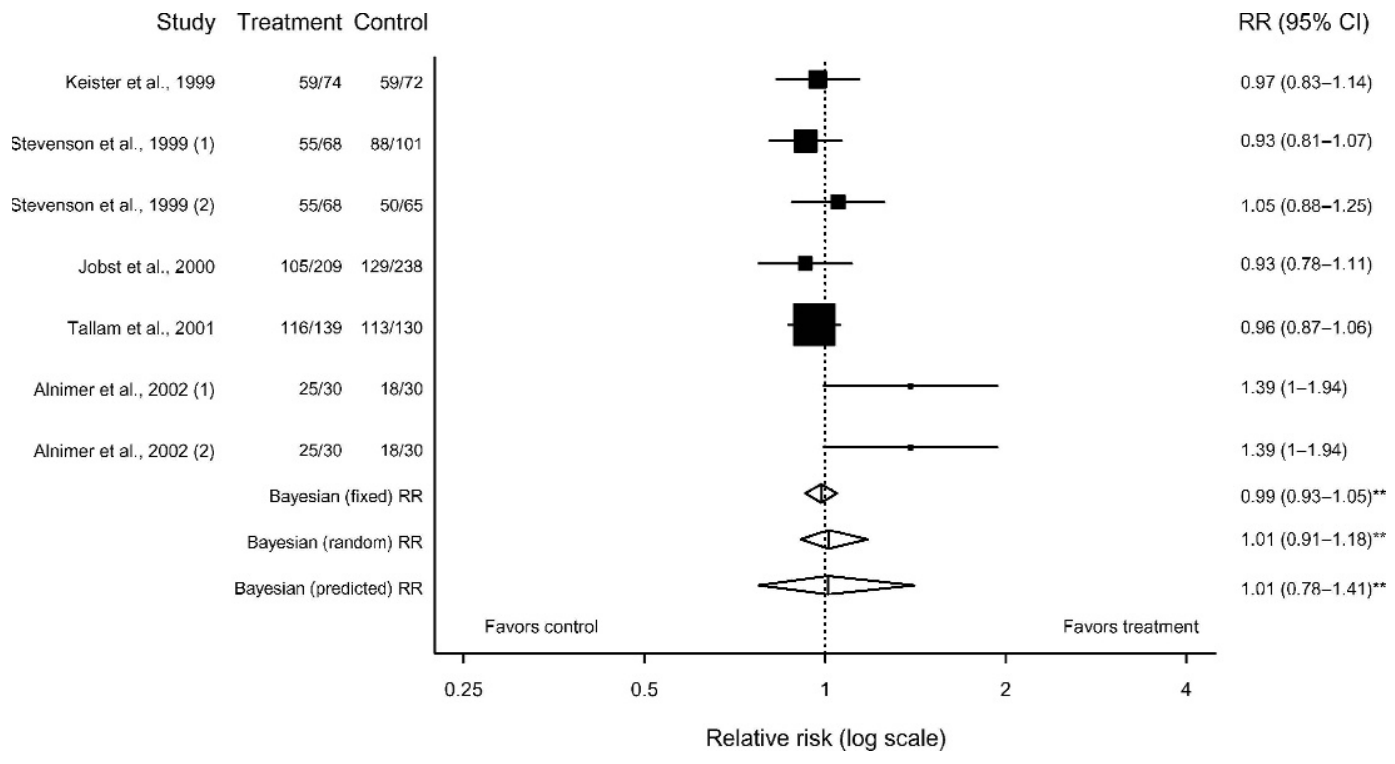

Figure 4. Relative risks (RR) [and their 95\% confidence interval (CI) for individual trials] determined from the results of 7 trials comparing overall pregnancy rates in Ovsynch and prostaglandin programs. Box sizes are proportional to the inverse variance of the estimates. Summary estimates of treatment effects are shown using: 1) a Bayesian (fixed effects) approach, 2) a Bayesian (random effects) approach, and 3) the predicted distribution of relative risk estimates expected in a future trial and their Bayesian credible intervals. Test of heterogeneity: $I^{2}=$ 45 (0-77); ** 95\% Bayesian credible interval. 


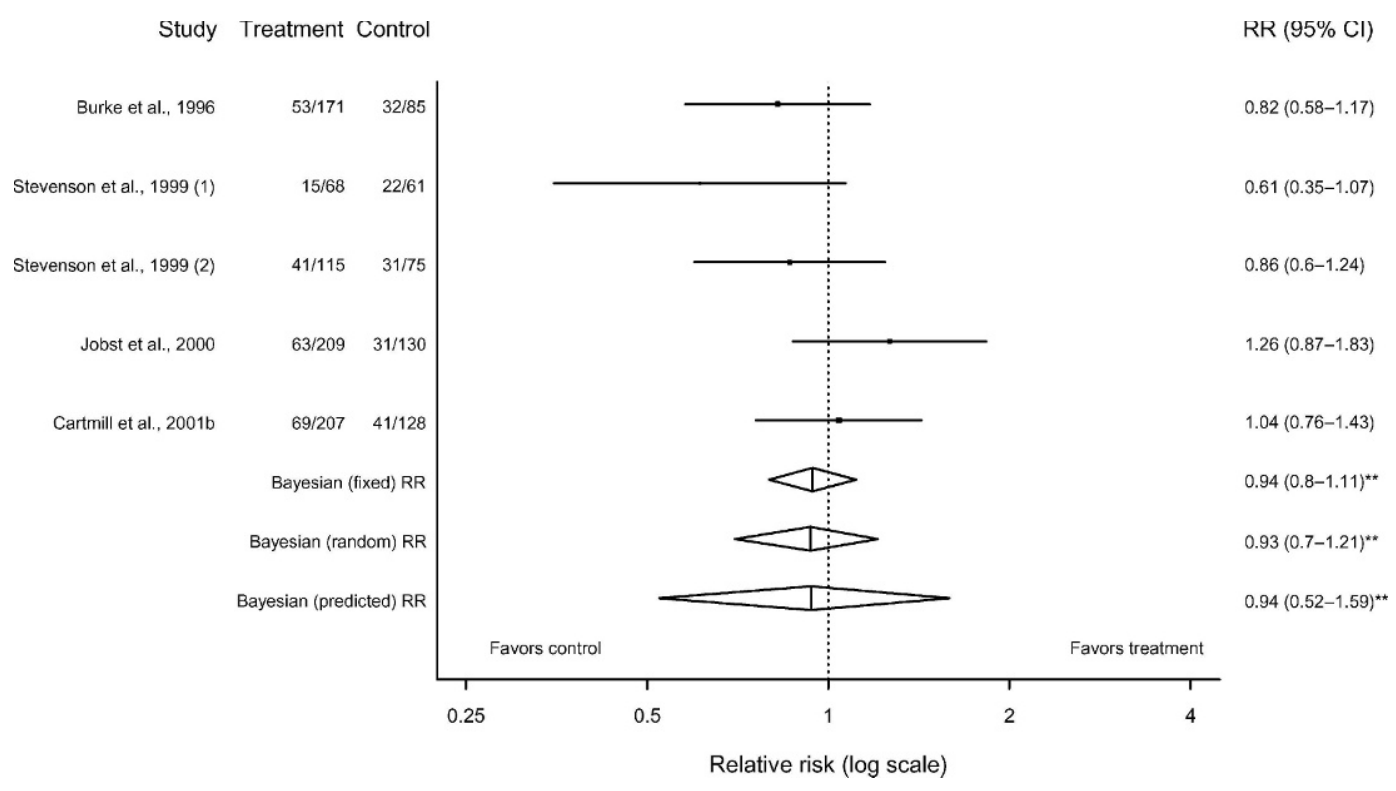

Figure 5. Relative risks (RR) [and their 95\% confidence interval (CI) for individual trials] determined from the results of 5 trials comparing conception rates in Ovsynch and Select Synch programs. Box sizes are proportional to the inverse variance of the estimates. Summary estimates of treatment effects are shown using: 1) a Bayesian (fixed effects) approach, 2) a Bayesian (random effects) approach, and 3) the predicted distribution of relative risk estimates expected in a future trial and their Bayesian credible intervals. Test of heterogeneity: $I^{2}=$ $34(0-75)$; test of heterogeneity following exclusion of studies marked with *: $I^{2}=0(0-76)$; ** 95\% Bayesian credible interval.

Study Treatment Control

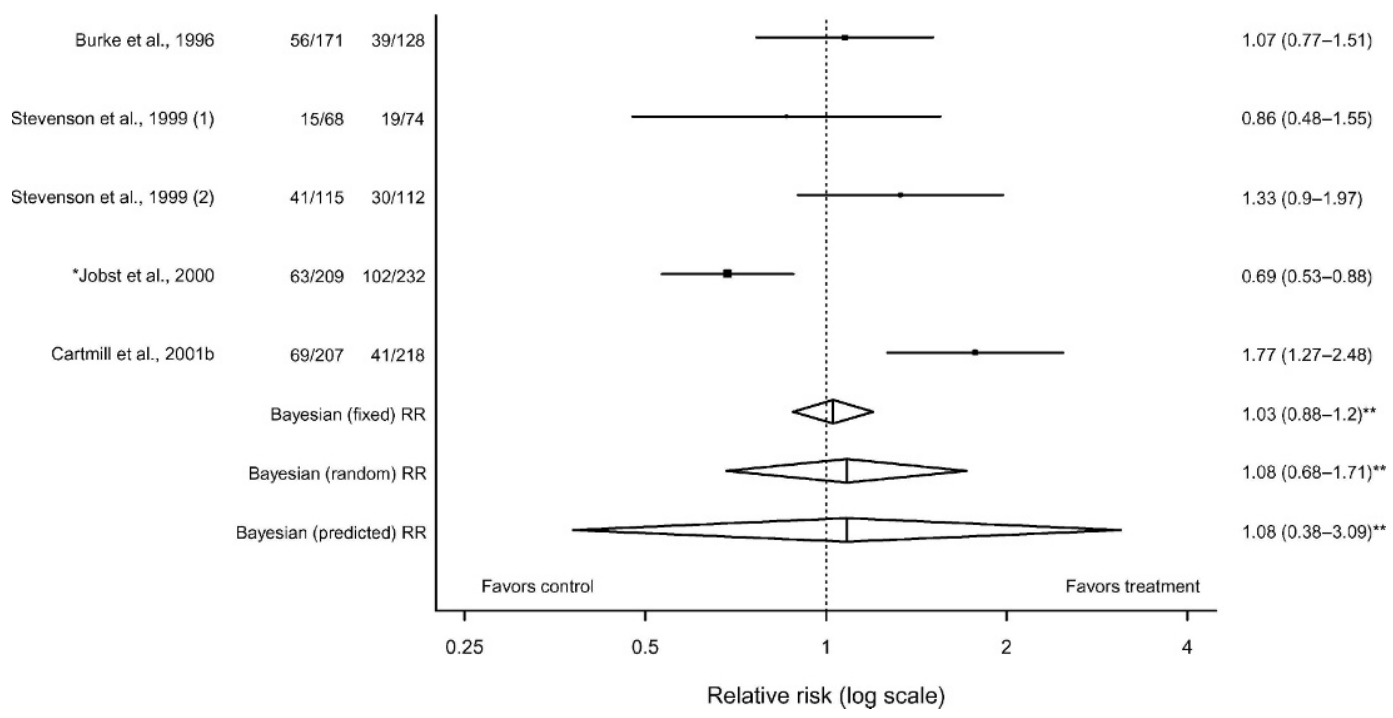

Figure 6. Relative risks (RR) [and their 95\% confidence interval (CI) for individual trials] determined from the results of 5 trials comparing pregnancy rates in Ovsynch and Select Synch programs. Box sizes are relative to the total number of animals in each trial. Summary estimates of treatment effects are shown using: 1) a Bayesian (fixed effects) approach, 2) a Bayesian (random effects) approach, and 3) the predicted distribution of relative risk estimates expected in a future trial and their Bayesian credible intervals. Test of heterogeneity: $I^{2}=$ 82 (60-92); test of heterogeneity following exclusion of studies marked with *: $I^{2}=52(0-84)$; ** 95\% Bayesian credible interval. 


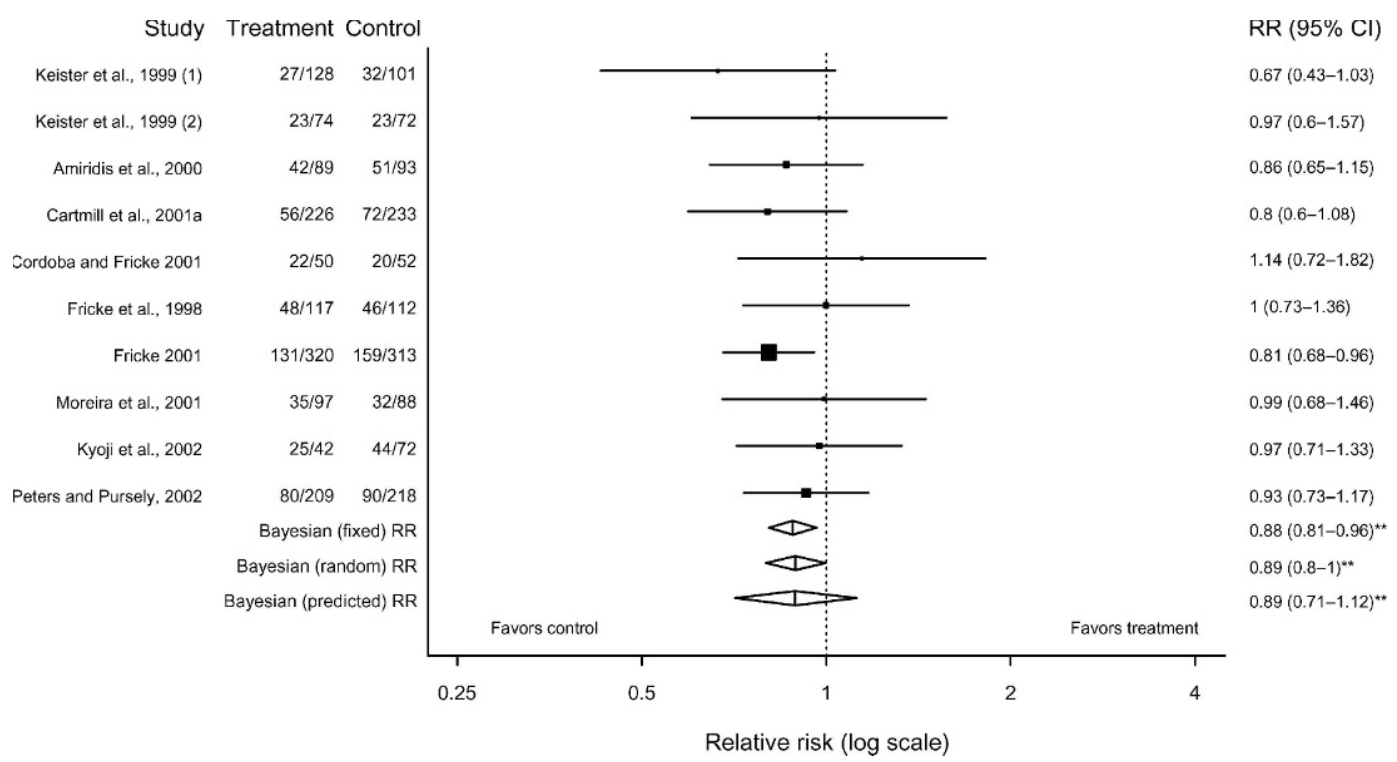

Figure 7. Relative risks (RR) [and their 95\% confidence interval (CI) for individual trials] determined from the results of 14 trials comparing pregnancy rates in Ovsynch and modified Ovsynch programs. Box sizes are proportional to the inverse variance of the estimates. Summary estimates of treatment effects are shown using: 1) a Bayesian (fixed effects) approach, 2) a Bayesian (random effects) approach, and 3) the predicted distribution of relative risk estimates expected in a future trial and their Bayesian credible intervals. Test of heterogeneity: $I^{2}=0(0-25) ; * * 95 \%$ Bayesian credible interval.

displays some evidence of a symmetrical funnel plot, suggestive of a lack of publication bias in this group of studies.

\section{DISCUSSION}

A wide range of reproductive technologies is available to producers to program reproductive management with the use of ovulation synchronization and timed

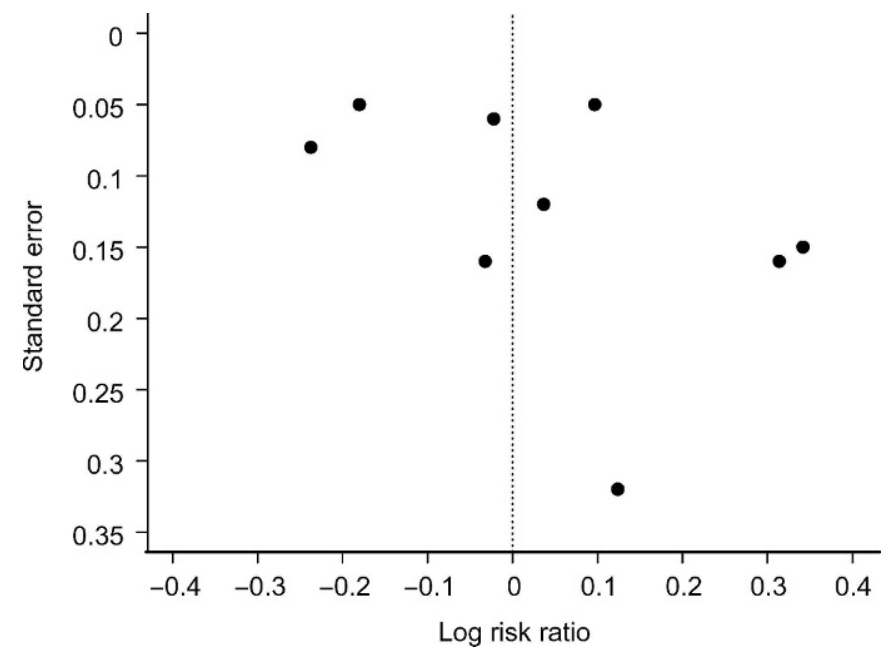

Figure 8. Funnel plot of log relative risk against standard error of conception rate for Ovsynch vs. natural breeding program. insemination protocols. Users of these technologies need to understand which of these programs are more practical, convenient, and effective. The Ovsynch/TAI protocol is a recent advance in reproductive biology that has been recommended to improve reproduction of lactating dairy cows. Any new technology or therapy must be evaluated under realistic conditions that reflect the complexities of interactions among management skills, milk production, environment, and nutrition. This

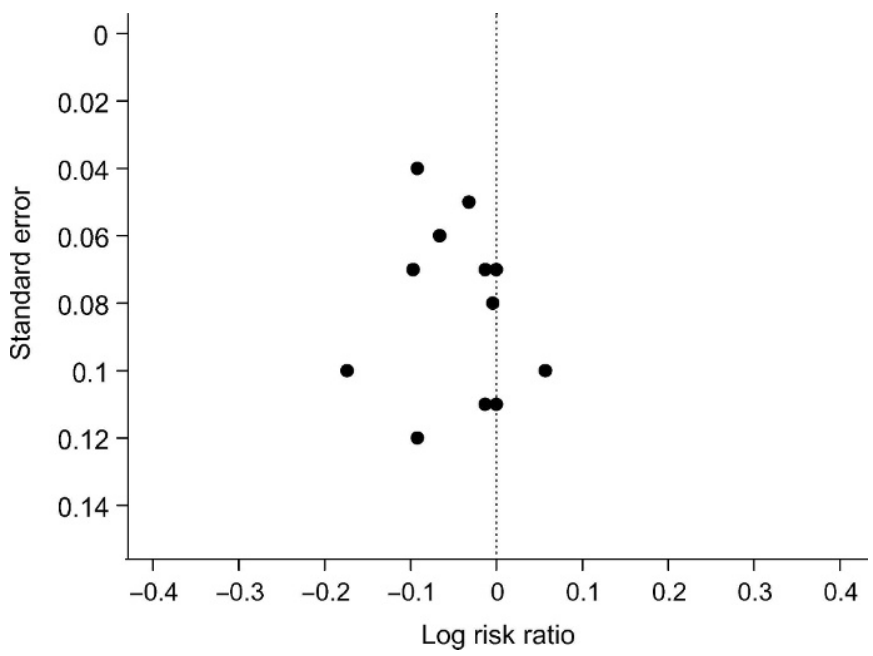

Figure 9. Funnel plot of log relative risk against standard error of conception rate for Ovsynch vs. modified Ovsynch program. 
study used meta-analytical techniques to compare the Ovsynch program with other synchrony programs, using studies conducted in a wide range of production systems.

Only papers that investigated and reported fertility data, such as conception and pregnancy rates, were included to evaluate the efficacy of programs to improve the reproductive management of the herd. Although other papers that reported the effect of Ovsynch program on follicular development and corpus luteum function are important, these studies did not provide fertility data and were not used. We intended to evaluate the time-associated fertility events (e.g., 21-d conception rates) for the trials included in this study. Those fertility measures would be ideal for use in meta-analysis. However, very few studies reported these time-associated incidences for pregnancy and conception rates; of those that reported these time-associated incidences, the time measured from the insemination to pregnancy differed. Most published papers referred to conception/ pregnancy to the first and second insemination or mating. Therefore, the conception and pregnancy rates as defined earlier in this paper were evaluated for each study and included in the analysis.

Determinations of the degree of similarity or difference between the results of apparently similar studies and examination of possible sources of variability are primary issues in the conduct of a meta-analysis (Greenland, 1994; Olkin, 1994). Consequently, we examined sources of heterogeneity in the study results. The relatively small number of trials from which data suitable for analysis were available in some cases resulted in relatively large predicted Bayesian CI of RR, and reduced our ability to draw more definite conclusions. Funnel plot analysis of results in this study showed that the results of a number of smaller trials, and trials with negative or positive outcomes of programs may not have been published. The plots showed little evidence of a funnel shape, were difficult to interpret, and reflected a need for more studies.

\section{Ovsynch vs. Natural Breeding}

Results of pregnancy rates for the Ovsynch and natural breeding programs did not differ significantly (predicted Bayesian $=1.04$, Bayesian CI $=0.36$ to 3.23, Figure 1, Table 2). Heterogeneity was influenced largely by the results of Cordoba and Fricke (2002) and Jobst et al. (2000), who reported lower pregnancy rates than other studies. There was no notable difference in study design between these and other trials included in the meta-analysis, except that in these studies, insemination was predominantly based on tail-paint removal (Cordoba and Fricke, 2002) and Heatwatch (Jobst et al., 2000) rather than visual observation. Sensitivity analyses conducted following the exclusion of these 2 studies (Jobst et al., 2000; Cordoba and Fricke, 2002) showed that the remaining studies were homogeneous $\left(I^{2}=34 \%\right.$, uncertainty intervals $=0$ to $\left.72 \%\right)$. In the natural breeding group, when inseminated at observed estrus, pregnancy rates were lower, and in some trials were comparable with the Ovsynch program. Insemination of cows based on the tail-paint removal method resulted in a greater pregnancy rate than those based on observed estrus. Therefore, the advantage of Ovsynch in increasing the number of cows submitted is diminished by the lack of a sensitivity of some heatdetection methods.

The homogeneity of the available trial data, following the removal of studies of Cordoba and Fricke, (2002) and Jobst et al. (2000), indicated that pregnancy rate was significantly improved in the Ovsynch group compared with those in the naturally bred group (Pursley et al., 1997b; Aréchiga et al., 1998; Keister et al., 1999). These data also suggest that reproduction of lactating dairy cows can be managed effectively without estrus detection by using the Ovsynch protocol.

\section{Ovsynch vs. PGF ${ }_{2 \alpha}$}

The Ovsynch and $\mathrm{PGF}_{2 \alpha}$ synchrony programs produced similar outcomes for conception and pregnancy data. The $\mathrm{PGF}_{2 \alpha}$ programs included single, double, or triple prostaglandin injections with some modifications in the breeding protocol. These studies were, therefore, not identical, and not conducted under similar conditions. For example, the studies of de la Sota et al. (1998) and Aréchiga et al. (1998) were conducted under summer heat conditions. However, we consider that the studies were sufficiently similar to be compared with a view to estimating measures of interest in the metaanalysis. The risk of conception and pregnancy did not differ significantly with cows synchronized with $\mathrm{PGF}_{2 \alpha}$ programs compared with those treated with Ovsynch protocol (Figures 2, 3, and 4).

In most of the $\mathrm{PGF}_{2 \alpha}$ program studies, the breeding programs were based on insemination at or after detected estrus, whereas in the Ovsynch program, all inseminations were based on TAI breeding protocol, regardless of estrus detection. Consequently, it could be expected that the Ovsynch program would produce greater pregnancy rates. However, there was not a significant improvement in the risk of pregnancy with the Ovsynch breeding program. In this study, all conception rate data extracted from $\mathrm{PGF}_{2 \alpha}$ trials were based on insemination at or after detected estrus, except one study (Stevenson et al., 1999, trial 2; Table 1). Stevenson et al. (1999) reported that conception rates were 
lower after TAI, but conception rates were greater when AI occurred after observed estrus. The pooled estimates in this study showed that conception and pregnancy rates were slightly and nonsignificantly better in cows treated with the Ovsynch protocol compared with those in the $\mathrm{PGF}_{2 \alpha}$ programs.

The results of studies reporting conception and pregnancy rates were heterogeneous (Table 2). Heterogeneity was influenced largely by the results of studies of Alnimer et al. (2002), Jemmeson et al. (2000), and Britt and Gaska (1998) for conception rate data, and de la Sota et al. (1998), Tenhagen et al. (2001), Alnimer et al. (2002), Britt and Gaska (1998) for pregnancy rate data. Sensitivity analyses conducted following the exclusion of these trials from the study showed that heterogeneity persisted in the remaining studies. This heterogeneity could not be readily attributed to consistent differences among trial protocols.

The risk of pregnancy by the end of breeding program (overall pregnancy) with the Ovsynch protocol was comparable with $\mathrm{PGF}_{2 \alpha}$ programs (Figure 4). The results of studies reporting the overall pregnancy were homogeneous $\left(I^{2}=45 \%\right.$, uncertainty intervals $=0$ to $77 \%$, Table 2). These results show that the Ovsynch program may be as effective as $\mathrm{PGF}_{2 \alpha}$ programs in improving pregnancy rates, although the Ovsynch protocol would be able to provide a very predictable time of AI after the start of treatment (Stevenson et al., 1996; Alnimer et al., 2002). These results show that the Ovsynch program could benefit dairy operations by allowing TAI of lactating cows without detection of estrus, producing similar pregnancy rates to those achieved by $\mathrm{PGF}_{2 \alpha}$ programs.

The PGF synchrony programs varied greatly; for example, some were single injections of PGF, and others were double or triple PGF programs. Injection intervals varied, as did breeding programs, for example, single AI vs. TAI vs. multiple AI. The approach used by the original authors of these papers in comparing some of these multiple AI PGF programs with the Ovsynch program, especially with a single treatment and only one AI, is questionable. We note the limitations of these approaches, and suggest that further meta-analyses with greater numbers of studies available may exclude some studies based on more equitable comparison.

\section{Ovsynch vs. Select Synch}

The risk of conception with Ovsynch treated cows did not differ from those treated with Select Synch protocols (predicted Bayesian RR $=0.94$, Bayesian CI RR $=0.52$ to 1.59 , Figure 5), and the results were homogeneous $\left(I^{2}=34 \%\right.$, uncertainty intervals $=0$ to $75 \%$, Table 2$)$. These data suggested that Select Synch programs could produce a similar conception rate to the Ovsynch program. However, the breeding program with Select Synch depends on estrus detection, which could be a practical disadvantage compared with the Ovsynch program.

The risk of pregnancy did not differ between Ovsynch cows and those treated with Select Synch protocols (predicted Bayesian $=1.08$, Bayesian CI $=0.38$ to 3.09). Heterogeneity in the results of studies reporting pregnancy rates $\left(I^{2}=82 \%\right.$, uncertainty intervals $=60$ to $92 \%$, Table 2) was influenced largely by the results of Jobst et al. (2000) who reported lower pregnancy rate with Ovsynch program than other trials. Differences in the study design between these studies and other trials were the dose and type of GnRH preparation used in the studies of Jobst et al. (2000). Sensitivity analyses showed that results of the remaining trials were homogeneous $\left(\mathrm{I}^{2}=52 \%\right.$, uncertainty intervals $=0$ to $84 \%$, Table 2). An improvement in the risk of pregnancy can be achieved with Ovsynch program if cows are inseminated on detected estrus (Cartmill et al., 2001a), a program similar to the Select Synch program breeding protocol. These data demonstrate that the reproductive performance of cows treated with Ovsynch and Select Synch protocols are comparable.

\section{Ovsynch vs. Modified Ovsynch}

The risk of conception and pregnancy in cows synchronized with modified Ovsynch did not differ from those in the Ovsynch program (predicted Bayesian $R R=$ 0.89 , Bayesian CI $=0.71$ to 1.12 , Figure 7 ), and the results of these studies were homogeneous $\left(I^{2}=0 \%\right.$, uncertainty intervals $=0$ to $25 \%$ ). A conception or pregnancy rate of individual studies using modified Ovsynch were comparable, and in some studies greater than an Ovsynch program. Most of studies we defined as modified Ovsynch were based on presynchronization of the estrus cycle, with single or double $\mathrm{PGF}_{2 \alpha}$ or an insertion of a controlled internal drug release device before the implementation of the Ovsynch protocol (Keister et al., 1999; Cartmill et al., 2001a; Fricke, 2001; Moreira et al., 2001). Small numbers of studies on each modification were a limitation and prevented analysis of these as separate groups. If the studies kept the core hormonal therapies of the Ovsynch program (GnRH$\mathrm{PGF}_{2 \alpha}$-GnRH) and similar timing of breeding with modification at either the beginning or end of the program, these were pooled to provide a single point estimate. Increased pregnancy rates were detected when cows that were presynchronized before the implementation of Ovsynch were compared with cows that had no presynchronization treatments (Figure 7). The likely reason for increased pregnancy rates in presynchronized 
cows was the increased frequency of cows initiating the synchronization protocol at favorable stages of the estrous cycle (Thatcher et al., 2001a). Effective resynchronization programs place cows between d 5 to 10 of the cycle at the time of the GnRH injection depending upon which day the cows expressed estrus after the injection of $\mathrm{PGF}_{2 \alpha}$. Days 5 to 10 of the cycle are considered the optimal time to begin the Ovsynch program (Thatcher et al., 2001a,b). These modifications to the Ovsynch program produced a comparable, and in some cases slightly higher, pregnancy rate compared with those treated with the Ovsynch program. The CoSynch program protocol (modified Ovsynch) requires cows to be handled only 3 times and, thus, would be easier to incorporate into an AI program. Therefore, modifications such as presynchronization of the Ovsynch protocol and altering the time of $\mathrm{AI}$ (CoSynch) could be suitable TAI programs for commercial dairy herds.

\section{CONCLUSIONS}

Bayesian pooled and predicted credible intervals obtained using the random-effect models were wider than the confidence intervals of the fixed models, providing more conservative estimates of each treatment effect that was evaluated. The point estimates derived from these studies from both fixed- and random-effect (pooled and predicted) models show that the conception and pregnancy rates obtained with the Ovsynch program were comparable with other synchrony techniques, but varied in effect among studies and with different programs.

Progress toward improving reproductive efficiency in lactating dairy cows may be achieved by combining TAI with a protocol for synchronization of ovulation that can be initiated at any stage of the estrous cycle. Overall, conception/pregnancy rates obtained with PGF, Select Synch, and modified Ovsynch programs were similar to the Ovsynch program. However, synchronization of ovulation using Ovsynch protocols can provide an effective way to manage reproduction in lactating dairy cows by eliminating the need for estrus detection. The modifications in Ovsynch program such as presynchronization and TAI at the time of second GnRH injection (CoSynch) can be a useful alternative for reproductive management of dairy herds with less than optimal estrus detection rates. Advantages of modified Ovsynch included data that are more homogeneous, greater submission rates than natural breeding, and avoidance of estrus detection. However, data on conception or pregnancy density (time-associated indices) are also required to allow the comparison of the efficacy of different synchrony programs.

\section{ACKNOWLEDGMENT}

We acknowledge and appreciate the information that was provided by some of the referenced authors for this study.

\section{REFERENCES}

Alnimer, M., G. De Rosa, F. Grasso, F. Napolitano, and A. Bordi. 2002. Effect of climate on the response to three oestrous synchronization techniques in lactating dairy cows. Anim. Reprod. Sci. 71:157168.

Amiridis, G. S., S. Belibasaki, L. Leontides, A. Lymberopoulos, and E. Aainas. 2000. Reproductive efficiency of three estrus synchronization schemes comprising fixed-time insemination in dairy cows. J. Vet. Med. A. 47:271-276.

Aréchiga, C. F., C. R. Staples, L. R. McDowell, and P. J. Hansen. 1998. Effects of timed insemination and supplemental $\beta$-carotene on reproduction and milk yield of dairy cows under heat stress. J. Dairy Sci. 81:390-402.

Bartolome, J. A., F. T. Silvestre, A. C. M. Arteche, S. Kamimura, L. F. Archbald, and W. W. Thatcher. 2002. The use of Ovsynch and Heatsynch forre-synchronization of cows open at pregnancy diagnosis by ultrasonography. J. Dairy Sci. 85(Suppl. 1):99. (Abstr.)

Britt, J. S., and J. Gaska. 1998. Comparison of two estrus synchronization programs in a large, confinement-housed dairy herd. JAVMA 212:210-212.

Burke, J. M., R. L. de la Sota, C. A. Risco, C. R. Staples, E. J.-P. Schmitt, and W. W. Thatcher. 1996. Evaluation of timed insemination using a gonadotropin-releasing hormone agonist in lactating dairy cows. J. Dairy Sci. 79:1385-1393.

Butler, W. R. 2000. Nutritional interactions with reproductive performance in dairy cattle. Anim. Reprod. Sci. 60-61:449-457.

Cartmill, J. A., S. Z. El-Zarkouny, B. A. Hensley, G. C. Lamb, and J. S. Stevenson. 2001a. Stage of cycle, incidence and timing of ovulation and pregnancy rates in dairy cattle after three timed breeding protocols. J. Dairy Sci. 84:1051-1059.

Cartmill, J. A., S. Z. El-Zarkouny, B. A. Hensley, T. G. Rozell, J. F. Smith, and J. S. Stevenson. 2001b. An alternative AI breeding protocol for dairy cows exposed to elevated ambient temperatures before or after calving or both. J. Dairy Sci. 84:799-806.

Cordoba, M. C., and P. M. Fricke. 2001. Evaluation of two hormonal protocols for synchronization of ovulation and timed artificial insemination in dairy cows managed in grazed-based dairies. J. Dairy Sci. 84:2700-2708.

Cordoba, M. C., and P. M. Fricke. 2002. Initiation of the breeding season in a grazing-based dairy by synchronization of ovulation. J. Dairy Sci. 85:1752-1763.

DeJarnette, J. M., and C. E. Marshall. 2003. Effects of pre-synchronization using prostaglandins $\mathrm{PGF}_{2 \alpha}$ and (or) $\mathrm{GnRH}$ on pregnancy rates of Ovsynch- and Cosynch-treated lactating Holstein cows. Anim. Reprod. Sci. 77:51-60.

DeJarnette, J. M., R. R. Salverson, and C. E. Marshall. 2001. Incidence of premature estrus in lactating dairy cows and conception rates to standing estrus or fixed-time inseminations after synchronization using $\mathrm{GnRH}$ and $\mathrm{PGF}_{2 \alpha}$. Anim. Reprod. Sci. 67:27-35.

de la Sota, R. L., J. M. Burke, C. A. Risco, F. Moreira, M. A. DeLorenzo, and W. W. Thatcher. 1998. Evaluation of timed insemination during summer heat stress in lactating dairy cattle. Theriogenology 49:761-770.

Domenici, F., G. Parmigiani, R. L. Wolpert, and V. Hasselblad. 1999.Meta-analysis of migraine headache treatments: Combining information from heterogeneous designs. J. Am. Stat. Assoc. 94:16-28.

Egger, M., and G. D. Smith. 2001. Principles of and procedures for systematic reviews. Pages 23-42 in Systematic Reviews in Health Care Meta-analysis in Context. M. Egger, G. D. Smith, and D. G. Altman, ed. British Medical Journal, London, UK. 
Fetrow, J., and T. Blanchard. 1987. Economic impact of the use of prostaglandin to induce estrus in dairy cows. JAVMA 190:163169.

Fricke, P. M. 2001. Ovsynch, Pre-synch, the Kitchen-Synch: What's up with synchronization protocols? Pages 91-102 in Proc. Midwest Dairy Herd Health Conference, Eau Claire, WI. University of Wisconsin-Madison, Eau Claire, WI.

Fricke, P. M., J. N. Guenther, and M. C. Wiltbank. 1998. Efficacy of decreasing the dose of GnRH used in a protocol for synchronization of ovulation and timed AI in lactating diary cows. Theriogenology 50:1275-1284.

Geary, T. W., J. C. Wittier, E. R. Downing, D. G. LeFever, R. W. Silcox, M. D. Holland, T. M. Nett, and G. D. Niswender. 1998. Pregnancy rates of postpartum beef cows that were synchronized using Syncro-Mate B or the Ovsynch protocol. J. Anim. Sci. 76:1523-1527.

Gelman, A. 1996. Inference and monitoring convergence. Pages 131143 in Markov Chain Monte Carlo in Practice. W. Gilks, S. Richardson, and D. Spiegelhalter, ed. Chapman and Hall, London, UK.

Greenland, S. 1994. Invited commentary: A critical look at some popular meta-analysis. Am. J. Epidemiol. 140:290-298.

Higgins, J. P. T., and S. G. Thompson. 2002. Quantifying heterogeneity in a meta-analysis. Stat. Med. 21:1539-1558.

Higgins, J. P. T., S. G. Thompson, J. J. Deeks, and D. G. Altman. 2003. Measuring inconsistency in meta-analysis. Br. Med. J. 327:557-560.

Jemmeson, A. 2000. Synchronising ovulation in dairy cows with either two treatments of gonadotropin-releasing hormone and one of prostaglandin, or two treatments of prostaglandin. Aust. Vet. J. 78:108-111.

Jobst, S. M., R. L. Nebel, M. L. McGilliard, and K. D. Pelzert. 2000. Evaluation of reproductive performance in lactating dairy cows with prostaglandin $\mathrm{F}_{2 \alpha}$, gonadotropin-releasing hormone, and timed artificial insemination. J. Dairy Sci. 83:2366-2372.

Keister, Z. O., S. K. DeNise, D. V. Armstrong, R. L. Ax, and M. D. Brown. 1999. Pregnancy outcomes in two commercial dairy herds following hormonal scheduling programs. Theriogenology 51:1587-1596

L'Abbe, K. A., A. S. Detsky, and K. O'Rouke. 1987. Meta-analysis in clinical research. Review. Ann. Intern. Med. 107:224-233.

Lean, I. J., J. A. Porter, A. R. Rabiee, W. F. Morgan, W. P. Tranter, N. Moss, and R. J. Rheinberger. 2003. Comparison of effects of $\mathrm{GnRH}$ and prostaglandin in combination, and prostaglandin on conception rates and time to conception in dairy cows. Aust. Vet. J. 81:488-493.

Light, R. J., and D. B. Pillemer. 1984. Summing up: The science of reviewing research. Harvard University Press, Cambridge, MA.

Mawhinney, I., H. Biggadike, and B. Drew. 1999. Field trial of a planned breeding regiment for dairy cows, using gonadotropinreleasing hormone and prostaglandin- $\mathrm{F}_{2 \alpha}$. Vet. Rec. 145:551-554.

Mialot, J. P., G. Laumonnier, C. Ponsart, H. Fauxpoint, E. Barassin, A. A. Ponter, and F. Deletang. 1999. Postpartum subestrus in dairy cows: Comparison of treatment with prostaglandin $\mathrm{F}_{2 \alpha}$ or $\mathrm{GnRH}+$ prostaglandin $\mathrm{F}_{2 \alpha}+\mathrm{GnRH}$. Theriogenology 52:901-911.

Moreira, F., C. Orlandi, C. A. Risco, R. Mattos, F. Lopes, and W. W. Thatcher. 2001. Effects of presynchronization and bovine somatotropin on pregnancy rates to a timed artificial insemination protocol in lactating dairy cows. J. Dairy Sci. 84:1646-1659.

Olkin, I. 1994. A critical look at some popular meta-analytic methods. Am. J. Epidemiol. 140:297-299.

Pancarci, S. M., E. R. Jordan, C. A. Risco, M. J. Schouten, F. L. Lopes, F. Moreira, and W. W. Thatcher. 2002. Use of estradiol cypionate in a presynchronized timed artificial insemination program for lactating dairy cattle. J. Dairy Sci. 85:122-131.

Pankowski, J. W., D. M. Galton, H. N. Erb, C. L. Guard, and Y. T. Grohn. 1995. Use of prostaglandin $\mathrm{F}_{2 \alpha}$ as a postpartum reproductive management tool for lactating dairy cows. J. Dairy Sci. 78:1477-1488.
Peters, M. W., and J. R. Pursley. 2002. Fertility of lactating dairy cows treated with Ovsynch after presynchronization injections of $\mathrm{PGF}_{2 \alpha}$ and GnRH. J. Dairy Sci. 85:2403-2406.

Peto, R. 1987. Why do we need systematic overviews of randomized trials? Stat. Med. 6:233-244.

Pursley, J. R., M. R. Kosorok, and M. C. Wiltbank. 1997a. Reproductive management of lactating dairy cows using synchronization of ovulation. J. Dairy Sci. 80:301-306.

Pursley, J. R., M. O. Mee, and M. C. Wiltbank. 1995. Synchronization of ovulation in dairy cows using $\mathrm{PGF}_{2 \alpha}$. Theriogenology 44:915-923.

Pursley, J. R., M. C. Wiltbank, J. S. Stevenson, J. S. Ottobre, H. A. Garverick, and L. L. Anderson. 1997b. Pregnancy rates per artificial insemination for cows and heifers inseminated at a synchronized ovulation or synchronized estrus. J. Dairy Sci. 80:295-300.

Quaife, T. 1995. Targeted breeding. Dairy Herd Management (June): 24-25.

Radostits, O. M., K. E. Leslie, and J. Fetrow. 1994. Herd health: Food animal production medicine. 2nd ed. W. B. Saunders Co., Philadelphia, PA

Raftery, A., and S. Lewis. 1992a. One long run with diagnostics: Implementation strategies for Markov chain Monte Carlo. Stat. Sci. 7:493-497.

Raftery, A., and S. Lewis. 1992b. How many iterations in the Gibbs sampler? Pages 763-774 in Bayesian Statistics 4. J. Benardo, J. Berger, A. Dawid, A. Smith, ed. Oxford University Press, London, UK.

Ryan, D. P., S. Snijders, H. Yaakub, and K. J. O’Farrell. 1995. An evaluation of estrus synchronization programs in reproductive management of dairy herds. J. Anim. Sci. 73:3687-3695.

Schmitt, É. J.-P., T. Diaz, M. Drost, and W. W. Thatcher. 1996. Use of a gonadotropin-releasing hormone agonist or human chorionic gonadotropin for timed insemination in cattle. J. Anim. Sci. 74:1084-1091.

Spiegelhalter, D. J., A. Thomas, N. G. Best, and W. R. Gilks. 2002 WinBUGS: Bayesian Inference Using Gibbs Sampling, version 1.4. Medical Research Council Biostatistics Unit, Cambridge, UK.

Stevenson, J. S., Y. Kobayashi, M. P. Shipka, and K. C. Rauchholz. 1996. Altering conception of dairy cattle by gonadotropin-releasing hormone preceding luteolysis induced by prostaglandin $\mathrm{F}_{2 \alpha}$. J. Dairy Sci. 79:402-410.

Stevenson, J. S., Y. Kobayashi, and K. E. Thompson. 1999. Reproductive performance of dairy cows in various programmed breeding systems including Ovsynch and combination of gonadotropin-releasing hormone and prostaglandin $\mathrm{F}_{2 \alpha}$. J. Dairy Sci. 82:506-515.

Stevenson, J. S., M. C. Lucy, and E. P. Call. 1987. Failure of timed inseminations and associated luteal function in dairy cattle after two injections of prostaglandin $\mathrm{F}_{2 \alpha}$. Theriogenology 28:937-946.

Stevenson, J. S., J. F. Smith, and D. E. Hawkins. 2000. Reproductive outcomes for dairy heifers treated with combinations of prostaglandin $\mathrm{F}_{2 \alpha}$, norgestomet and gonadotropin-releasing hormone. J. Dairy Sci. 83:2008-2015.

Tallam, S. K., T. L. Kerbler, K. E. Leslie, K. Bateman, W. H. Johnson, and J. S. Walton. 2001. Reproductive performance of postpartum dairy cows under a highly intervenient breeding program involving timed insemination and combinations of GNRH, prostaglandin $\mathrm{F}_{2 \alpha}$ and human chorionic gonadotropin. Theriogenology 56:91-104

Tenhagen, B. A., M. Drillich, and W. Heuwiser. 2001. Analysis of cow factors influencing conception rates after two timed breeding protocols. Theriogenology 56:831-838.

Thatcher, W. W., R. Mattos, F. Moreira, M. Binelli, and J. D. Ambrose. 2000. Experimental manipulation of follicular growth. Reprod. Domest. Anim. (Suppl. 6):27-33.

Thatcher, W. W., F. Moreira, S. M. Pancarci, J. A. Bartolome, and J. E. P. Santos. 2002. Strategies to optimize reproductive efficiency by regulation of ovarian function. Domest. Anim. Endocrinol. 23:243-254. 
Thatcher, W. W., F. Moreira, J. E. P. Santos, R. C. Mattos, F. L. Lopes, S. M. Pancarci, and C. A. Risco. 2001b. Effects of hormonal treatments on reproductive performance and embryo production. Theriogenology 55:75-89.

Thatcher, W. W., D. J. Patterson, F. Moreira, M. Pancarci, and E. R. Jordan. 2001a. Current concepts for estrus synchronization and timed insemination. 34th Ann. Proc. Am. Soc. Bovine Pract. 34:96-105.

Twagiramungu, H., L. A. Guilbault, and J. J. Dufour. 1995. Synchronization of ovarian follicular waves with a gonadotropin-releasing hormone agonist to increase the precision of estrus in cattle. A Review. J. Anim. Sci. 73:3141-3151.

Whitehead, A. 2002. Selection bias. Pages 198-213 in Meta-analysis of Controlled Clinical Trials. John Wiley and Sons Ltd., London, UK.

Williams, S. W., R. L. Stanko, M. Amstalden, and G. L. Williams. 2002. comparison of three approaches for synchronization of ovulation for timed artificial insemination in Bos indicus-influenced cattle managed on the Texas gulf coast. J. Anim. Sci. 80:11731178 . 